\title{
A fork in the Sagittarius trailing debris
}

\author{
C. Navarrete ${ }^{1,2,3 \star}$, V. Belokurov ${ }^{3}$, S. E. Koposov ${ }^{3}$, M. Irwin ${ }^{3}$, M. Catelan ${ }^{1,2}$, \\ S. Duffau ${ }^{2,1}$ and A. J. Drake ${ }^{4}$ \\ ${ }^{1}$ Instituto de Astrofísica, Pontificia Universidad Católica de Chile, Av. Vicuña Mackenna 4860, 782-0436 Macul, Santiago, Chile \\ ${ }^{2}$ Millennium Institute of Astrophysics, Av. Vicuña Mackenna 4860, 782-0436, Santiago, Chile \\ ${ }^{3}$ Institute of Astronomy, University of Cambridge, Madingley Road, Cambridge, CB3 0HA, UK \\ ${ }^{4}$ Cahill Center for Astronomy and Astrophysics, California Institute of Technology, Pasadena, CA 91125
}

Last updated $2016 \mathrm{xxxx} \mathrm{xx}$; in original form $2016 \mathrm{xxxx} \mathrm{xx}$

\begin{abstract}
We take advantage of the deep and wide coverage of the VST ATLAS survey to study the line-of-sight structure of the Sagittarius stellar stream in the Southern hemisphere, only $\sim 40^{\circ}$ away from the progenitor. We use photometrically selected Sub-Giant Branch (SGB) stars to reveal a complex debris morphology of the trailing arm and detect at least two clear peaks in the SGB distance modulus distribution. The separation between the two line-of-sight components is at least $5 \mathrm{kpc}$ at the edge of the VST ATLAS footprint, but appears to change along the stream, which allows us to conclude that these detections correspond to two physically independent stellar structures, rather than a mix of co-distant stellar populations within a single stream. Our discovery of a fork in the Sgr trailing arm is verified using Blue Horizontal Branch stars and our distance measurements are calibrated using RR Lyrae stars from the Catalina Real-Time Transient Survey. Comparing with numerical simulations of the Sgr dwarf disruption, the more distant of the two components in the fork matches perfectly with the track of the trailing debris. However, no obvious counterpart exists in the simulation for the closer line-of-sight component.
\end{abstract}

Key words: Galaxy: halo - galaxies: individual (Sagittarius) - stars: horizontal branch

\section{INTRODUCTION}

More than a decade has passed since the vast expanse of the Sagittarius (Sgr) tidal stream was uncovered by Majewski et al. (2003) through dexterous data-mining of the 2MASS stellar catalogs. While the M-giants used in that study are perfectly suitable as a tracer to map out the extent of the stream, the full complexity of this structure remained underappreciated until more numerous and less metallicity-biased tracers were deployed. Main Sequence (MS) and Main Sequence Turn-Off (MSTO) stars outnumber M-giants by several orders of magnitude and thus can help to uncover lower level sub-structures within the tails. To that end, using the Sloan Digital Sky Survey (SDSS) Data Release 5 (DR5) multi-band photometry, Belokurov et al. (2006) showed that around the North Galactic cap the Sgr leading tail is bifurcated, i.e. split into two distinct components on the sky, a bright and a faint one, running alongside each other in distance. As the SDSS progressed from DR5 to DR9, a more complete view of the stream was put together by Koposov

^ Contact e-mail: cnavarre@astro.puc.cl et al. (2012), unveiling a similar bifurcation in the trailing tail in the Southern Galactic hemisphere. This was later confirmed by Slater et al. (2013) in an independent study using data from the Pan-STARRS1 survey. We note that both Koposov et al. (2012) and Slater et al. (2013) successfully used Red Clump (RC) stars as distance indicators in their studies of the stream.

The final SDSS footprint has left two large gaps in the Sgr stream coverage: one, at least $100^{\circ}$ wide in the immediate neighborhood of the progenitor, and one of a similar size in the direction towards the Galactic anti-center where the stream crosses the disk. Therefore, in these two regions, studies of the stream have so far relied on 2MASS M-giants. Unfortunately these comparatively metal-rich and relatively young stars may have been fogging the view of the Sgr tails. For example, the second, fainter component of the Sgr bifurcation does not appear to be traced by M-giants at all, which could be due to the metallicity differences between the two branches as explained in Koposov et al. (2012). Additionally, M-giants are not very useful distance indicators as their intrinsic luminosity strongly depends on both age and metallicity. Instead, as shown by Belokurov et al. (2006), 

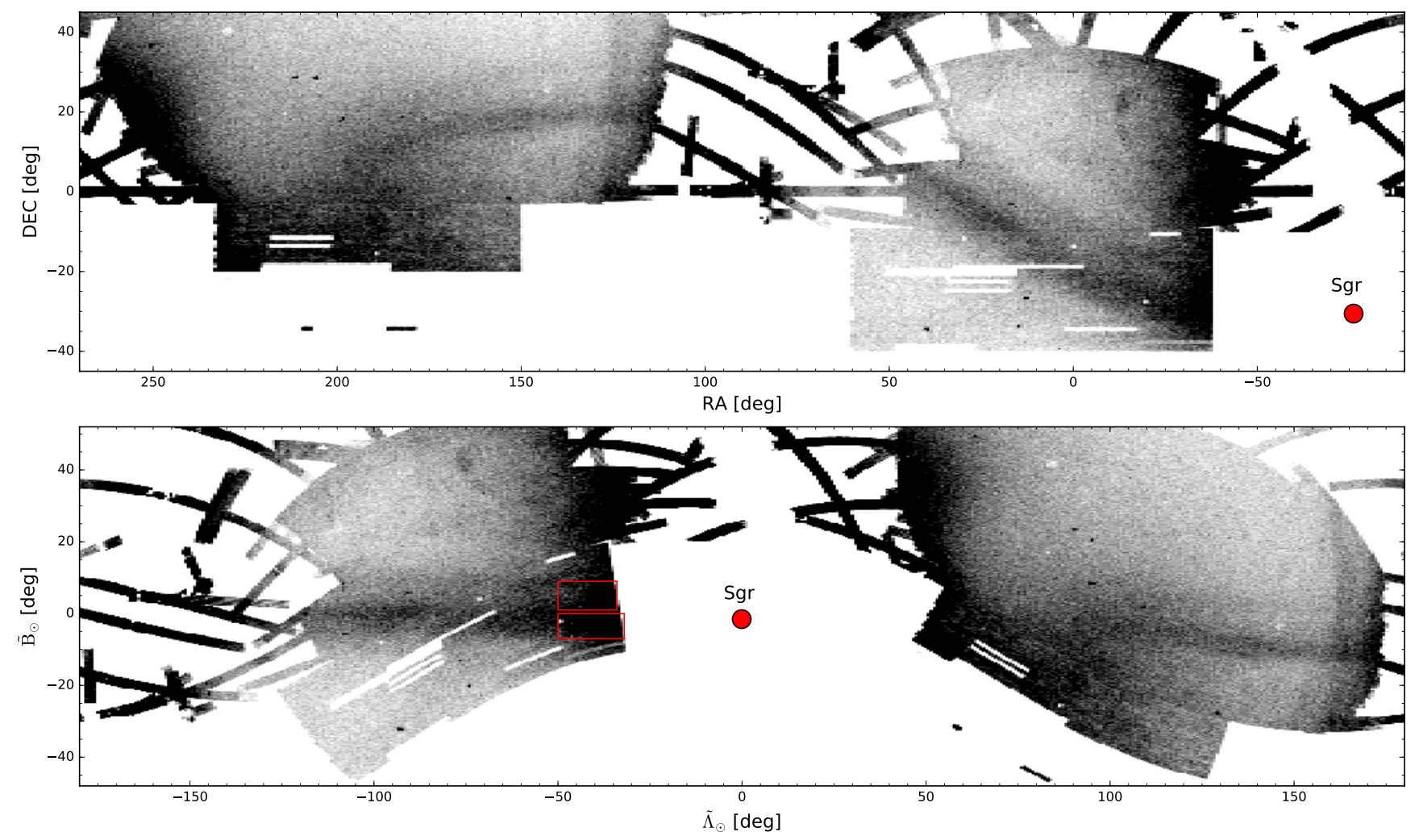

Figure 1. Density map of MSTO stars from the SDSS DR9 and VST ATLAS datasets, in equatorial (top) and the Sgr stream (bottom) coordinate systems. The darker regions correspond to enhanced stellar density. The red boxes enclose the area used to study the line of sight sub-structure on the Southern extension of the Sgr stream, for the bright $\left(\right.$ at $\left.\tilde{\mathbf{B}}_{\odot}<0^{\circ}\right)$ and faint $\left(\tilde{\mathbf{B}}_{\odot}>0^{\circ}\right)$ branches.

Sub-Giant Branch (SGB) stars can be selected to provide accurate relative distances, which can be then calibrated using less numerous but more trustworthy Blue Horizontal Branch (BHB) stars (see e.g. Yanny et al. 2009; Ruhland et al. 2011; Belokurov et al. 2014) or RR Lyrae (see e.g. Drake et al. 2013b).

As the Sun is located very close to the plane of the Sgr stream, the information on the shape of the tidal tails and thus the orbit of the progenitor is encoded in the run of the line-of-sight distances along the stream. Therefore, high distance accuracy is required to facilitate an unbiased gravitational potential inference. However, results so far have lead to some curious conclusions. This is illustrated in Law \& Majewski (2010) who find that only a triaxial Dark Matter (DM) halo resembling a hockey puck and oriented perpendicular to the Galactic disk can explain the distances along the Sgr leading arm. Another example is the study of Gibbons et al. (2014) who show that a very light Milky Way is needed to reproduce the Sgr stream orbital precession data obtained by Belokurov et al. (2014). Moreover, good distance precision is necessary to unpick multiple wraps of the stream in a given direction. For example, Belokurov et al. (2006) detected multiple debris components along the sightlines towards the leading tail using SGB stars as tracers. It is likely that the so-called $\mathrm{C}$ branch of the stream they see behind the leading debris is in fact a part of the trailing tail. Correnti et al. (2010) used RC stars to produce further evidence for multiple wraps of the stream.
While difficult to track down, stream wraps offer powerful leverage in tidal tail modelling. To begin with, they allow the minimal bound on the stream length to be improved. As shown in Erkal et al. (2016b), the stream length is a function of the progenitor mass, the time of disruption and the properties of the host gravitational potential. Thus, if the progenitor original mass is constrained independently (see e.g. Niederste-Ostholt et al. 2010) and the time since the beginning of disruption is deduced based on e.g. star-formation activity in the stream (de Boer et al. 2015), then the radial profile of the Galactic total density can be inferred. Typically, the fainter wraps of the stream are also the oldest detectable tidal debris. These, therefore, have accumulated the largest amount of differential orbital plane precession. According to Erkal et al. (2016a), this is manifested in both the width of the stream on the sky as well as the angular offset of the "wrapped" portion of the stream with respect to the dynamically younger debris. The plane precession is caused by the torques provided by the non-spherical portion of the Galactic force field. Therefore, through the analysis of the older stream wraps, a picture of the 3D shape of the Milky Way DM halo can be reconstructed.

In this paper, we analyze the most recent imaging data from the VST ATLAS survey, which covers a portion of the Sgr trailing tail in the Southern hemisphere not previously observed by any wide-field optical survey. In particular, we focus on the 3D behaviour of this un-studied part of the stream and show that i) the trailing tail bifur- 

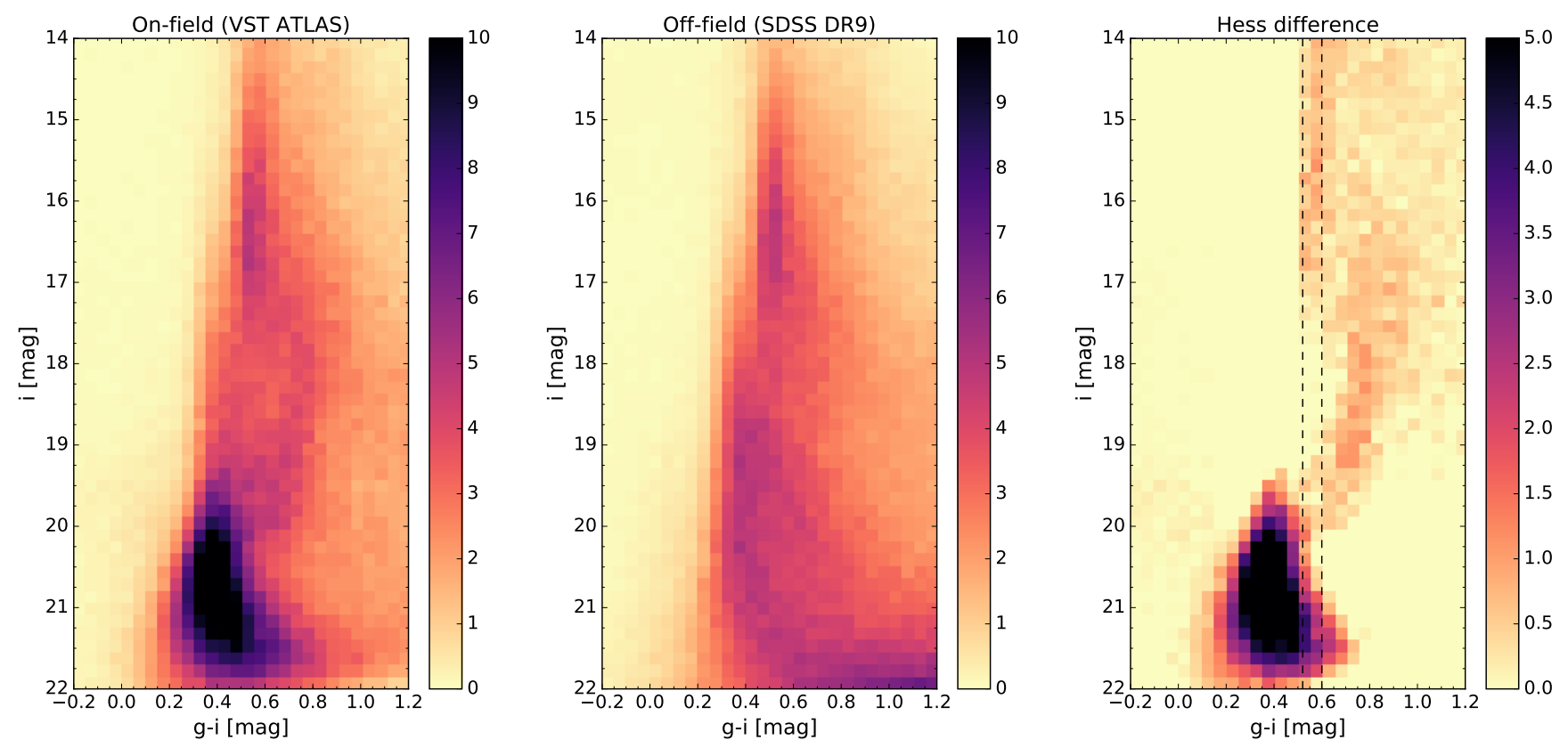

Figure 2. Left: Hess diagram for the on-stream field defined by $-50^{\circ}<\tilde{\Lambda}_{\odot}<-32^{\circ}$ and $-7^{\circ}<\tilde{\mathrm{B}}_{\odot}<0^{\circ}$. The MS and the MSTO of the Sgr population are both clearly visible at $i \sim 20$ mag and fainter. Middle: Hess diagram for the off-stream field, from SDSS data. Right: Background-subtracted Hess diagram of the Sgr stream. The MS, subgiant and red giant branches are all clearly recovered. The bin size in the Hess diagrams are $0.15 \mathrm{mag}$ in $i$ magnitude and 0.05 in $(g-i)$ color. The color bar scale corresponds to the number of stars per bin per square degree in each panel.
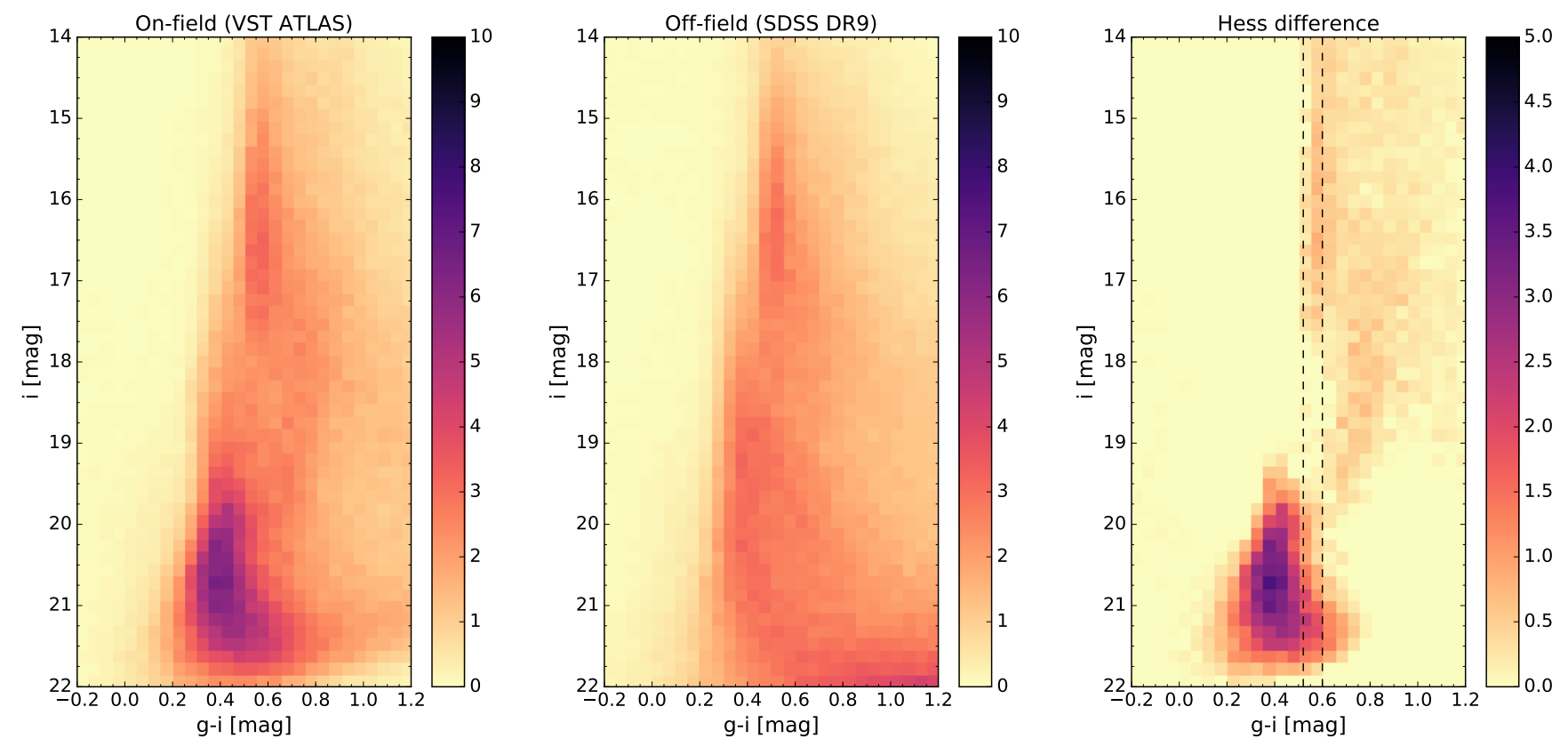

Figure 3. Same as Figure 2, but for the region enclosed by $-50^{\circ}<\tilde{\Lambda}_{\odot}<-34^{\circ}$ and $1^{\circ}<\tilde{\mathrm{B}}_{\odot}<9^{\circ}$, i.e. corresponding to the location of the faint branch of the Sgr trailing arm. The Hess difference hints at the existence of two SGBs, as in the bright branch, but with much less signal (compare with the right panel of Figure 2). 

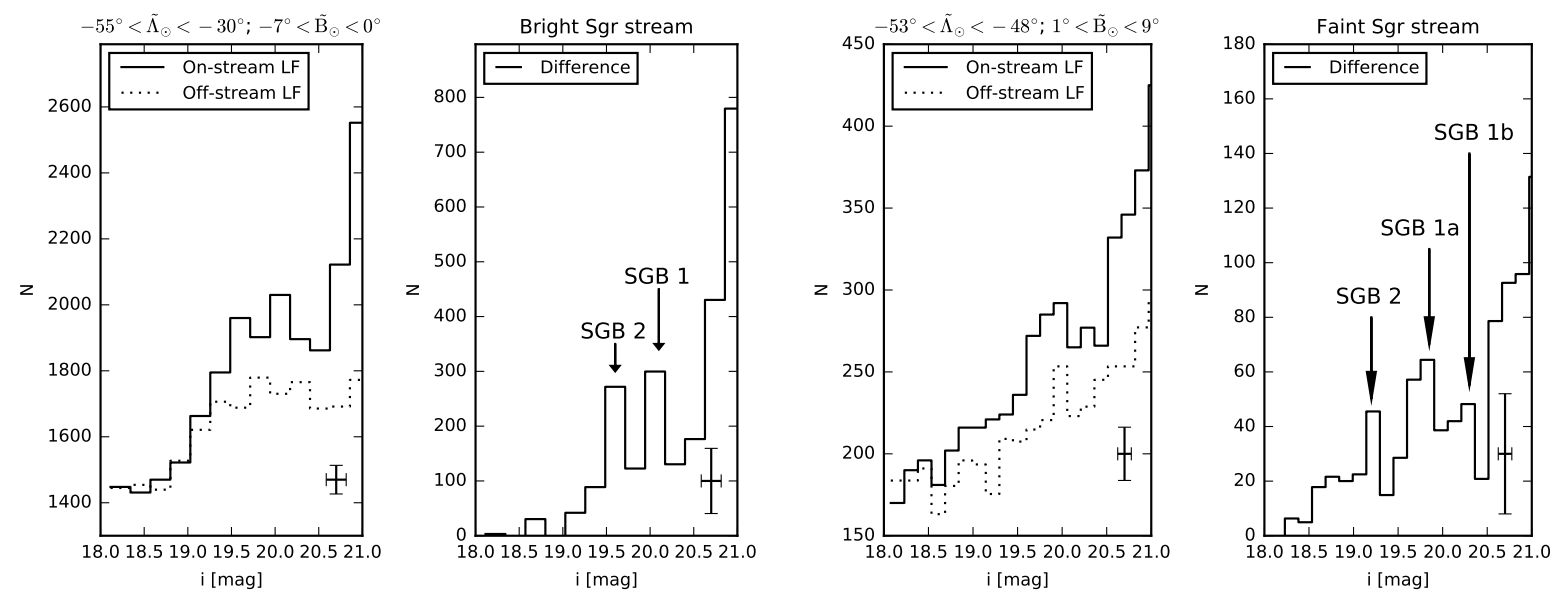

Figure 4. Luminosity functions of SGB stars selected to lie in a narrow color range in the bright branch (dashed lines in Figures 2 and 3). Left: Luminosity functions for the SGB stars for the on-stream field (solid line) and the off-stream field (dotted line), scaled by the ratio of their areas for the bright $\mathrm{Sgr}$ arm, at $-55^{\circ}<\tilde{\Lambda}_{\odot}<-30^{\circ}$ and $-7^{\circ}<\tilde{\mathrm{B}}_{\odot}<0^{\circ}$. Left middle: Difference of the luminosity functions for the on- and the off-stream fields shown in the left panel. Note the two peaks visible at $i \sim 19.6$ and 20.0 mag. Right middle: The same as leftmost panel, but for the area enclosing the Sgr faint branch: $-53^{\circ}<\tilde{\Lambda}_{\odot}<-48^{\circ}$ and $1^{\circ}<\tilde{\mathrm{B}}_{\odot}<9^{\circ}$. The distributions corresponds to the on-stream (solid line) and off-stream (dotted line) fields. Right: Difference of the luminosity functions for the on-stream region of the Sgr faint branch. Here three peaks are noticeable, at $i \sim 19.3,19.9$ and 20.3 mag. Representative error bars are shown at the bottom right in each panel.

cation detected by Koposov et al. (2012) continues into the VST ATLAS footprint and ii) the trailing debris appears to be split along the line-of-sight, with the additional stream component following a distinct distance track. This paper is structured as follows. Section 2 describes the VST ATLAS data and presents Color-Magnitude Diagrams (CMD) of the Sgr trailing tail. Section 3 presents the analysis of the CMD and the resulting relative distance measurements. The absolute distance scale is introduced in Section 3.2. Discussion and Conclusions can be found in Section 4.

\section{THE SGR STREAM IN THE ATLAS SURVEY}

The ATLAS survey (Shanks et al. 2015) is being carried out with the $2.6 \mathrm{~m}$ VLT Survey Telescope (VST) at ESO's Cerro Paranal Observatory in Chile. The survey uses the OmegaCAM camera, containing 32 CCDs of $4 \mathrm{k} \times 2 \mathrm{k}$ pixels with a sampling of $0.21^{\prime \prime}$ per pixel giving a field-of-view coverage of $1 \mathrm{deg}^{2}$. The ATLAS survey aims to cover $4700 \mathrm{deg}^{2}$ of the southern sky in five photometric passbands, ugriz, reaching depths similar to the SDSS (e.g., the $5 \sigma$ source detection limit is $23.1 \mathrm{mag}$ for the $g$ band). Basic image reduction and initial catalogue generation are provided by the Cambridge Astronomical Survey Unit (CASU) using the VST data flow software (for details see Shanks et al. 2015). The band-merging and selection of primary sources were performed as separate steps using a local SQL database (as described in detail in Koposov et al. 2014). Note that the VST ATLAS photometry used in this work has been calibrated with the data from AAVSO Photometric All-Sky Survey (APASS, Henden et al. 2016). While approximately similar to the SDSS, in practice the APASS gri filters do not match the SDSS ones exactly. Moreover, APASS does not provide any $u$-band data, therefore our calibration of the VST ATLAS $u$-band magnitudes relies on the $g$ and $r$ photometry. In this work, all magnitudes are dereddened using the Schlegel et al. (1998) extinction maps, adopting the extinction coefficients of Schlafly \& Finkbeiner (2011).

Figure 1 shows the spatial density distribution of MSTO stars in the SDSS DR9 (Ahn et al. 2012) and the VST ATLAS surveys stitched together, both in equatorial (top panel) and Sagittarius-stream (bottom panel) coordinates ${ }^{1}$. The MSTO stars were selected to have $0.0<(g-i)<0.6$ and $19<r<21 \mathrm{mag}$ in both datasets. The southern fingers of SDSS DR9, which overlap the observations of VST ATLAS, were excluded to not artificially increase the number of stars in those regions. VST ATLAS covers two big patches of the sky at negative declinations, centered at $(\alpha, \delta)=\left(195.0^{\circ}\right.$, $\left.-24.5^{\circ}\right)$; and $\left(10.0^{\circ},-11.0^{\circ}\right)$.

In the SDSS data, the two branches of the leading tail are visible crossing most of the SDSS footprint. The ATLAS survey observes the region of the Southern sky closer to the Sgr dwarf, thus extending the view of the trailing arm beyond what is available in the SDSS DR9 data. Note that Koposov et al. (2012) and Slater et al. (2013) had already identified two branches in the trailing tail in the South, similar to what is seen in the leading tail in the North. The VST ATLAS data unambiguously confirms the existence of the trailing bifurcation at lower $\left|\tilde{\Lambda}_{\odot}\right|$ as evidenced by the bottom panel of the Figure. To recap, the bifurcation consists of two branches that are separated on the sky by $\sim 10^{\circ}$. Note that, according to Slater et al. (2013), there could also be a difference in the heliocentric distances to the two branches, of order $\sim 5 \mathrm{kpc}$. The bulk of the "bright stream" is below the Sgr orbital plane (i.e., at $\tilde{\mathrm{B}}_{\odot}<0^{\circ}$ ) while the "faint stream" lies mostly above the plane $\left(\tilde{\mathrm{B}}_{\odot}>0^{\circ}\right)$. While providing some additional information on the density evolution of

$1\left(\tilde{\Lambda}_{\odot}, \tilde{\mathrm{B}}_{\odot}\right)$ are the coordinates of the Sgr orbital plane, as defined by Belokurov et al. (2014). 
the faint branch, the VST ATLAS covers mostly the region with $\tilde{\mathbf{B}}_{\odot}<0^{\circ}$. Thus the focus of this study is mostly on the bright branch of the trailing Sgr tail.

\subsection{Color-magnitude diagrams}

As a first diagnostic of the stellar populations in the Sgr stream as viewed by the VST ATLAS, let us inspect the Hess diagram of the portion of the trailing tail closest to the progenitor. More precisely, the CMD density distribution is constructed for stars in a region covering $-7^{\circ}<\tilde{\mathrm{B}}_{\odot}<0^{\circ}$ and $-50^{\circ}<\tilde{\Lambda}_{\odot}<-32^{\circ}$ (lower red box in Figure 1). Assuming that the Milky Way is roughly symmetric about the Galactic plane, we select an equivalent region (i.e. the same range of Galactic longitude, but positive Galactic latitude) in the North to be used as a representative of the background Milky Way population. Given that ATLAS does not observe the Northern sky, we take the photometry available from the SDSS DR9. Note that the ATLAS and the SDSS photometric systems do not match exactly, and therefore there may be magnitude offsets between the Hess diagrams, of the order of 0.03 mag.

Figure 2, from left to right, shows the Hess diagram for the on-stream field, that for the mirror patch of the sky and the difference of the two. The left and middle Hess diagrams were divided by the area subtended on the sky by each region, and therefore each bin represents the number of stars per magnitude per color, and per square degree. The Sgr population is evident already in the on-stream Hess diagram, even before the subtraction of the background. In the background-subtracted diagram, the MS, the sub-giant and the red giant branches can now be clearly seen. When the left panel of Figure 2 is compared to the third panel of Figure 4 of Belokurov et al. (2006), it is immediately apparent that the SGB portion of the Hess diagram of the trailing tail in the ATLAS data is much broader than the SGB of the leading tail in the SDSS. In fact, there is a striking resemblance of the behavior of the SGB considered here and the split SGB shown in the left panel of Figure 4 of Belokurov et al. (2006). In the North, the double SGB arises due to the projection along the line of sight of the two independent Sgr streams, the so-called branches A and C. We conjecture that a similar situation is observed in this portion of the trailing tail in the South: the possible existence of at least two, vertically offset populations is betrayed by the fatness of the MSTO and the thickness of the sub-giant and the red giant branches.

We have also examined the Hess diagram of the faint trailing tail, i.e. the portion of the sky with $1^{\circ}<\tilde{\mathbf{B}}_{\odot}<9^{\circ}$ and $-50^{\circ}<\tilde{\Lambda}_{\odot}<-34^{\circ}$ (upper red box in Figure 1). Figure 3 shows the on-stream Hess diagram, the off-stream Hess diagram, and the difference between the two. As in the previous case, the Hess diagrams are divided by the area covered by each region. The Sgr population is recovered as well, but it is much less prominent compared to the bright stream. Intriguingly, this branch of the stream - studied earlier by Koposov et al. (2012) and Slater et al. (2013) - also does not possess a particularly tight SGB, thus hinting at multiple debris along the line of sight in the direction of the faint trailing Sgr branch.

To confirm the existence of the two populations offset in magnitude, the luminosity functions (LF), i.e. the one- dimensional slices for the on- and off-stream fields can be directly compared in Figure 4. Based on the Hess diagram, we select SGB stars in the color range $0.52<(g-i)<0.6$ mag (dashed vertical lines in Figure 2 and 3). According to Figure 4, where the leftmost panel shows the on-stream LF (solid line) and the off-stream LF (dashed line, scaled to the ratio of areas between the two fields), the typically rather narrow SGB region is much broader than expected, spanning as much as 1 mag. Note that the steep rise of the luminosity function in the on-stream data from $i=20.5 \mathrm{mag}$ onwards is mostly due to the stream MS stars. The difference between the luminosity functions (left middle panel) shows two distinct peaks at $i=19.6$ and $20.0 \mathrm{mag}$, with the faintest one being the most prominent of the two. Given that the SGB peaking at the fainter magnitude contains more stars, we choose to designate it SGB 1, and its counterpart, peaking $~ 0.6$ mag brighter, SGB 2. Using the off-stream LF as a model of the background, the significance of SGB 1 and SGB 2 is 6.1 and $5.3 \sigma$, respectively.

In the case of the faint Sgr stream, the on-stream LF (right middle panel) also shows a broad SGB peak around $i \sim 19.8 \mathrm{mag}$. The subtraction of the off-stream field (from SDSS data) shows the presence of three peaks at $i \sim 19.3$, 19.9 and 20.4 mag. However, in this case, the number counts are much less than for the Sgr bright stream. In fact, the significance of these peaks is much lower: SGB 1a has $3.0 \sigma$ of significance, while the secondary peak (dubbed SGB 1b) has only $1.5 \sigma$. SGB 2 also has a low significance, namely $\sim 2 \sigma$. Despite being hardly statistically significant, the detections are also recovered with other tracers (see Section 3.3), which suggests they might indeed be real substructures, but less prominent than the two streams found in the bright trailing arm (above the Sgr's plane).

The presence of two peaks in the SGB luminosity function of the bright $\mathrm{Sgr}$ stream (i.e., $\tilde{\mathrm{B}}_{\odot}<0^{\circ}$ ), separated by about $0.6 \mathrm{mag}$, could be either due to two distinct Sgr tidal debris overdensities at two different distances along the line of sight, or, alternatively, due to a complex stellar population in the Sgr stream, albeit all at the same distance. Another possibility that cannot be ruled out is that one of the two detected populations corresponds to an entirely new stream, not connected to the Sgr dwarf in any way. In what follows, we shall trace the position of the stars in the two SGB peaks as a function of the longitude along the Sgr coordinate system, in an attempt to elucidate the genesis of the split SGB. The same analysis is performed for the faint Sgr tail. However, as mentioned before, the core of this work is the analysis of the SGB detections on the bright Sgr tail; while the analysis of the faint one should be considered as a first attempt to trace the density evolution of this less populated signature.

\section{STREAM TOMOGRAPHY}

\subsection{Exposing the Sagittarius SGB}

Figure 5 shows the density of the candidate SGB stars with $-7^{\circ}<\tilde{\mathrm{B}}_{\odot}<0^{\circ}$ in the space of $i$ magnitude and $\tilde{\Lambda}_{\odot}$. Here, a much larger extent of the Sgr longitude is studied, i.e. $-100^{\circ}<\tilde{\Lambda}_{\odot}<-30^{\circ}$, corresponding approximately to the entire additional stream coverage supplied by the ATLAS 

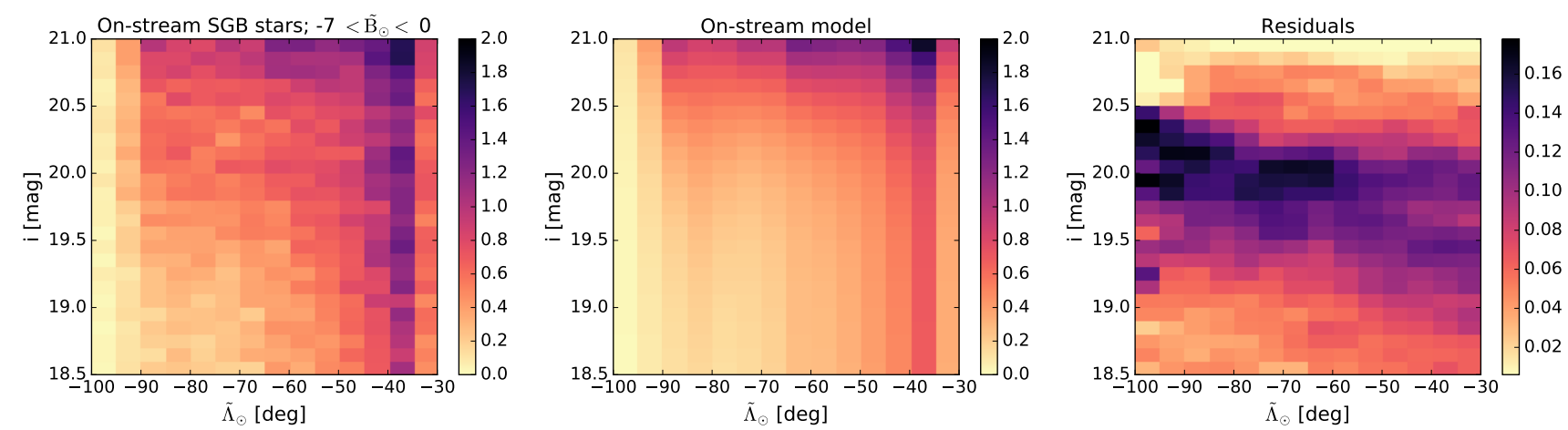

Figure 5. Density of the bright branch SGB stars as a function of the longitude along the stream, $\tilde{\Lambda}_{\odot}$, and the $i$-band magnitude. The on-stream field (left), the model (center) and the residuals (right) are shown. The $2 \mathrm{D}$ density was divided by the area covered by the field. The diagrams are $14 \times 25$ pixels, and the residuals are smoothed with a Gaussian kernel with a FWHM of 0.7 pixels. At each $\tilde{\Lambda}_{\odot}$ bin, the residuals are vertically normalized. The two peaks in the Sgr bright stream (the "fork") start at $\tilde{\Lambda}_{\odot} \sim-30^{\circ}$, separated by $\sim 0.6$ $\mathrm{mag}$, and tend to merge at $\tilde{\Lambda}_{\odot} \sim-70^{\circ}$. Afterwards, there are also two peaks, with the most prominent one located at i 20.2 mag, and another much less prominent at $\sim 19.4$ mag.
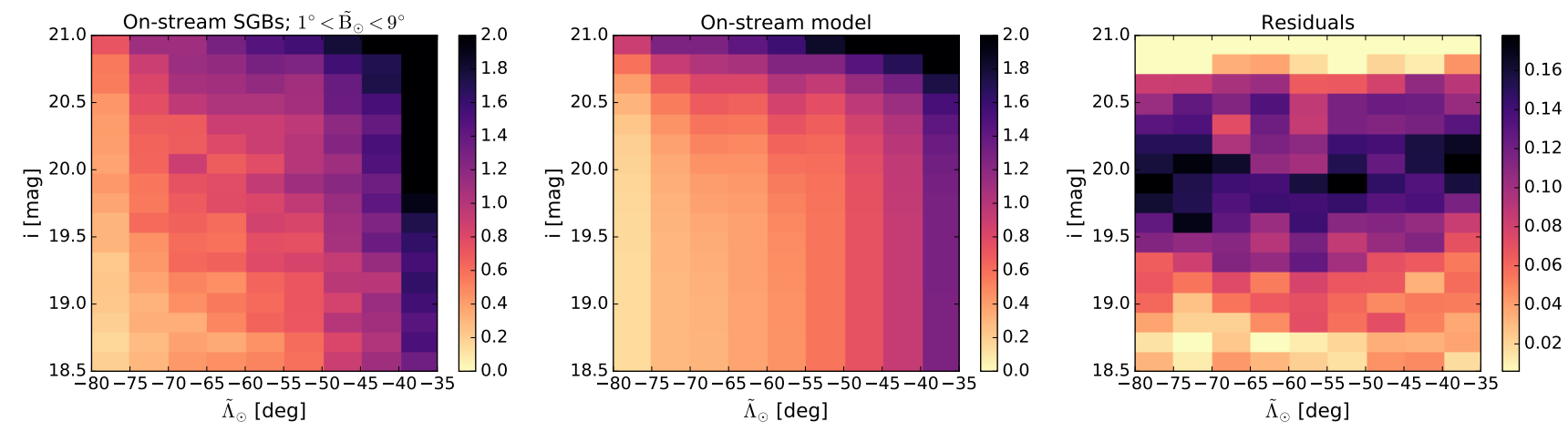

Figure 6. Same as Figure 5, but now for the SGB stars in the region between $1^{\circ}<\tilde{\mathrm{B}}_{\odot}<9^{\circ}$, i.e. the so-called faint branch. At each $\tilde{\Lambda}_{\odot}$ bin, the residuals are vertically normalized. The diagrams are $9 \times 17$ pixels, and the residuals are smoothed with a Gaussian kernel with a FWHM of 0.7 pixels In this case, the signal of SGB stars is most prominent at $i \sim 20$ mag, with a secondary peak at $i \sim 20.5$ mag. In some $\tilde{\Lambda}_{\odot}$ bins, a small increase in the number of SGB stars is found at $i \sim 19$ mag.
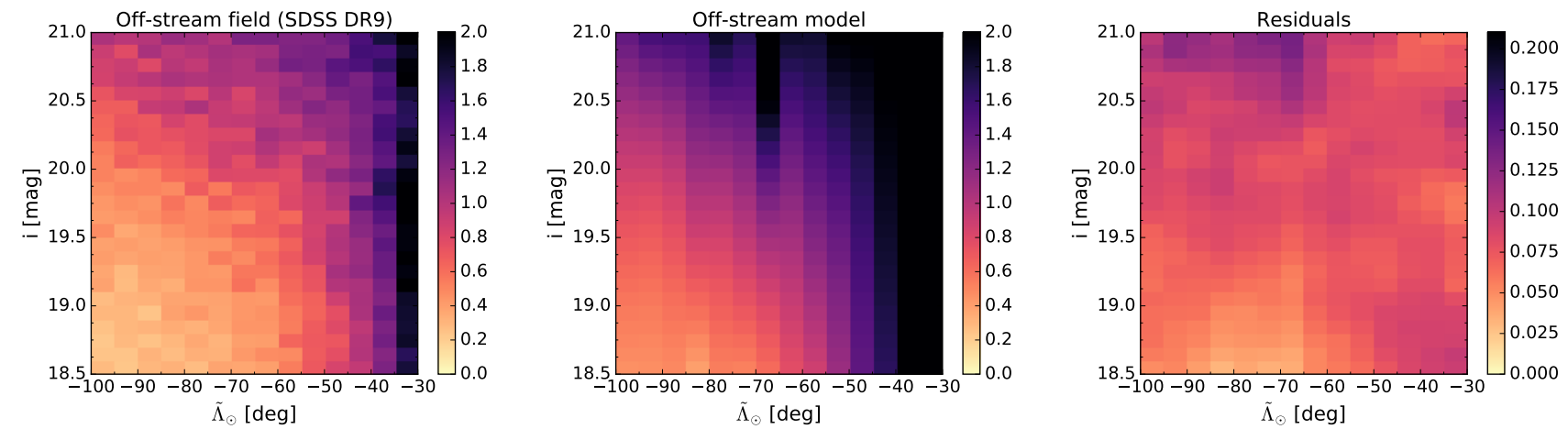

Figure 7. Same as Figure 5, but now for the SGB stars in the off-stream test field (covered by the SDSS DR9). The off-stream field (left), the model (center) and the residuals (right) are shown. At each $\tilde{\Lambda}_{\odot}$ bin, the residuals are vertically normalized and the residuals were smoothed using a Gaussian kernel with a FWHM of 0.7 pixels. No positive residuals can be traced in the magnitude range of interest, as expected for an area without any known halo sub-structure (with distances similar to those of the Sgr stream). 

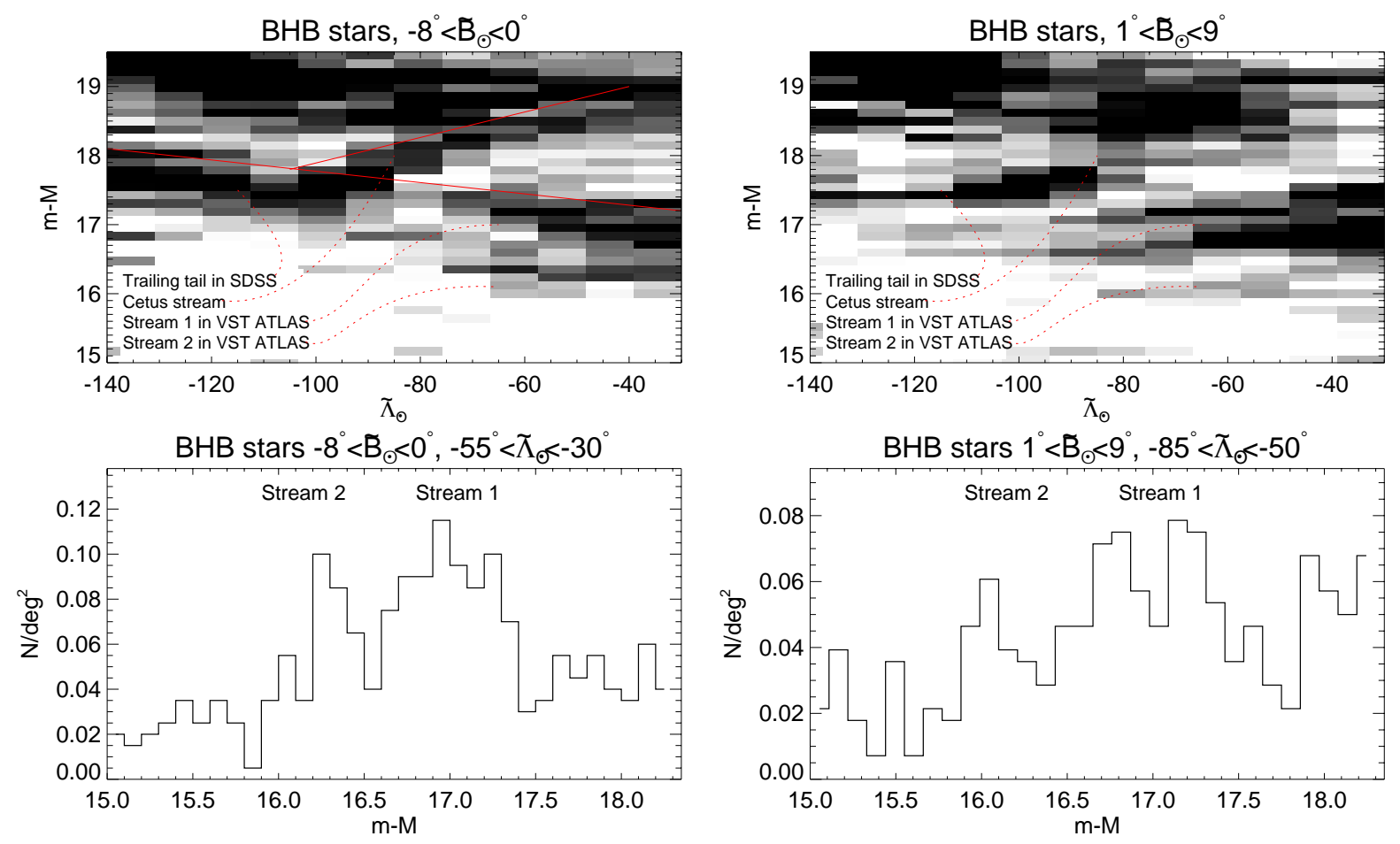

Figure 8. Line-of-sight distribution of BHB candidate stars. Top: Greyscale density of the BHB candidate stars in the plane of distance modulus vs. Sgr stream longitude $\tilde{\Lambda}_{\odot}$ for the bight (left) and the faint (right) trailing arm branches. The darker regions correspond to an enhanced stellar density. Note that a combination of BHB candidate stars from both SDSS and VST ATLAS datasets is shown here. The main structures, including the Sgr and the Cetus Polar Stream debris, are marked. The two solid lines show the distance gradient of the Sgr and the Cetus streams. Bottom: Slices through the $2 \mathrm{D}$ BHB density plots shown in the Top panel for a small range of $\tilde{\Lambda}_{\odot}$ (as indicated in the title of each panel). Peaks corresponding to different line-of-sight components of the trailing arm are clearly visible (see main text for a detailed discussion).

survey. In order to trace the variation (if any) in the magnitude of the two SGB peaks along the Sgr longitude, the stream region is divided into 14 segments, each 5 degrees long, while keeping the vertical bins at $0.1 \mathrm{mag}$. In the left panel of the Figure, two tendencies are immediately apparent: the steep "vertical" rise of the star counts, i.e. larger densities at fainter magnitudes due to i) the increased halo contribution with growing volume probed and ii) increased contribution from the MS stars in the Sgr stream; as well as the "horizontal" density increase to lower $\left|\tilde{\Lambda}_{\odot}\right|$ due to the drop in the Galactic latitude. Note that the abrupt decrease in the density at $\tilde{\Lambda}_{\odot}>-40^{\circ}$ is due to the incompleteness of the ATLAS footprint. It is evident that the stream SGB signal is buried under the strong density gradients in both the magnitude and longitude dimensions.

To reveal the Sgr stream SGB, rather than trying to identify an appropriate patch of the sky for comparison, we put forward the following simple model of the background star counts. In each bin of $\tilde{\Lambda}_{\odot}$, the luminosity function is approximated with an exponential profile $N(m a g)=A \exp (B \times$ $m a g)+C$. We allow the model parameters $A, B$ and $C$ to vary from bin to bin along $\tilde{\Lambda}_{\odot}$, and mask out the magnitude range of interest, i.e. pixels with $18.8<i<20.2$ (see Figure 4). The middle panel of Figure 5 shows the variation of the model luminosity function obtained.

The right panel of Figure 5 shows the distribution of the residuals after subtraction of the model described above from the data. In order to improve the visibility of the over- densities along each line of sight, this $2 \mathrm{D}$ histogram is vertically normalized, i.e. the counts in each $\tilde{\Lambda}_{\odot}$ bin are divided by the total in each column and the binsize. The SGB signal is now clearly discernible between $i=19$ and $i=20.5$. From right to left, i.e. from lower $\left|\tilde{\Lambda}_{\odot}\right|$ values to higher, a shallow apparent magnitude gradient is visible, with the stream detectable at slightly fainter magnitudes around $\tilde{\Lambda}_{\odot}=-100^{\circ}$ compared to $\tilde{\Lambda}_{\odot}=-30^{\circ}$. The SGB magnitude gradient is due to the slow evolution of the heliocentric distance to the Sgr stream with $\tilde{\Lambda}_{\odot}$ as measured previously (see e.g. Koposov et al. 2012; Slater et al. 2013) and in agreement with the model of the dwarf disruption (see Section 4). Additionally, the width of the SGB band appears to evolve with the Sgr longitude. Finally, in agreement with the analysis reported in the previous Section, in the range $-60^{\circ}<\tilde{\Lambda}_{\odot}<-30^{\circ}$ two individual SGB components are visible. These are separated by $\sim 0.6$ mag at $\tilde{\Lambda}_{\odot} \sim-30^{\circ}$ and (nearly) merge together at $\tilde{\Lambda}_{\odot} \sim-70^{\circ}$.

Analogously, Figure 6 shows the behavior of the candidate $\mathrm{SGB}$ stars with $1^{\circ}<\tilde{\mathrm{B}}_{\odot}<9^{\circ}$, i.e. in the area corresponding to the faint Sgr branch. As in the previous Figure, the panels show the on-stream field (left), the on-stream model (middle) and the vertically-normalized residuals (right). The stream region was divided into 5 degrees long bins while the vertical bins were increased up to $0.15 \mathrm{mag}$, but the vertically-normalized residuals are also divided by the binsize, in order to reach the same signal level as in Figure 5. In this case, to construct the model the bins in the range 19.0 
Table 1. Distance modulus to the trailing SGB 1 and 2 peaks along the bright branch of the Sgr stream.

\begin{tabular}{lcccc}
\hline \hline $\begin{array}{c}\tilde{\Lambda}_{\odot} \\
\left(^{\circ}\right)\end{array}$ & $\begin{array}{c}\mathrm{m}-\mathrm{M}(\mathrm{SGB} 1) \\
(\mathrm{mag})\end{array}$ & $\begin{array}{c}\sigma(\mathrm{m}-\mathrm{M}) \\
(\mathrm{mag})\end{array}$ & $\begin{array}{c}\mathrm{m}-\mathrm{M}(\mathrm{SGB} 2) \\
(\mathrm{mag})\end{array}$ & $\begin{array}{c}\sigma(\mathrm{m}-\mathrm{M}) \\
(\mathrm{mag})\end{array}$ \\
\hline-97.5 & 17.20 & 0.10 & 16.74 & 0.15 \\
-92.5 & 17.10 & 0.07 & 16.71 & 0.15 \\
-87.5 & 17.09 & 0.06 & 16.52 & 0.16 \\
-82.5 & 17.01 & 0.10 & 16.85 & 0.07 \\
-77.5 & 17.05 & 0.04 & & \\
-72.5 & 16.87 & 0.11 & 16.71 & 0.07 \\
-67.5 & 16.92 & 0.06 & 16.48 & 0.12 \\
-62.5 & 16.78 & 0.15 & 16.63 & 0.12 \\
-57.5 & 16.80 & 0.05 & 16.31 & 0.06 \\
-52.5 & 16.84 & 0.05 & 16.44 & 0.10 \\
-47.5 & 16.82 & 0.16 & 16.52 & 0.12 \\
-42.5 & 16.87 & 0.03 & 16.28 & 0.03 \\
-37.5 & 16.96 & 0.13 & 16.37 & 0.17 \\
-32.5 & 16.80 & 0.07 & 16.29 & 0.11 \\
\hline
\end{tabular}

$<i<20.5$ mag were excluded. The residuals show a strong detection of an overdensity at similar apparent magnitude as compared to that in the bright branch. However, here the SGB signal displays a slightly different behavior with $\tilde{\Lambda}_{\odot}$, and, overall, the line-of-sight distribution of the SGB stars is more complicated, varying from narrow to broad, and in some directions showing what can be interpreted as two or even three distinct peaks.

In the following sub-section we measure the positions of the SGB peaks along the line of sight and convert apparent magnitudes into heliocentric distances. Before that, however, we perform a simple test to ensure the robustness of the analysis. As a sanity check, we apply the luminosity function modeling analysis described above to an SDSS field with equatorial coordinates $\left(190^{\circ}, 47^{\circ}\right)$, i.e. a location in the Galaxy where no obvious stellar halo overdensities have been detected to date in the distance range of interest. Figure 7 shows the density of the candidate SGB stars in the SDSS selected field with the same arrangement of panels as in the previous two figures. Interestingly, the density variations in the first two panels (data and model) appear to resemble those in the bright and faint streams shown in Figures 5 and 6. However, reassuringly, in the third panel no strong positive residuals are visible, thus giving us confidence that the signal revealed in the previous two figures is genuine.

\subsection{Distances to the Sgr trailing tail with SGB stars}

With the Sgr SGB signal now cleansed of the contamination, we fit a Gaussian mixture model to the residual density distributions shown in the right panels of Figures 5 and 6 to obtain the centroids of the SGB peaks. To get an idea of the systematic uncertainty of our peak centroiding procedure, we change the magnitude bins by $\pm 50 \%$ and re-measure the peak positions. The difference in the positions of each peak obtained with three different magnitude bin sizes is taken as representative of the systematic error in the method. At each Sgr longitude, the error bars for each peak are the sum (in quadrature) of the random errors on the peak determination from the Gaussian fit and the systematic error associated to the $i$ magnitude bin size. The same procedure is adopted to recover the centroids of the peaks in the Sgr faint stream.

The errors in distance modulus listed do not represent the possible dispersion in distance modulus along the line of sight. For each of the SGB detections, the dispersion of the distribution could be gleaned from the $\sigma$ of the Gaussian fit. For the faint arm detections, the three peaks have similar dispersions, generally lower than 0.2 mag. In the case of the detection in the bright arm, the dispersions are of the order of $0.3 \mathrm{mag}$. There is no evident trend of the peak as a function of $\tilde{\Lambda_{\odot}}$, neither the heliocentric distance with the associated dispersion along the line of sight. The same is not true for the RR Lyrae stars (see Section 3.4), which reveal a marked difference in heliocentric distance dispersion at different $\tilde{\Lambda_{\odot}}$.

The absolute distance calibration for the SGB peaks thus measured can be obtained using previous detections of the stream at the same $\tilde{\Lambda}_{\odot}$, based on tracers with reliable distances. The distances to the Sgr debris at $\tilde{\Lambda}_{\odot}=-97.5^{\circ}$ and $-92.5^{\circ}$ were previously measured by Koposov et al. (2012), using the RC stars $^{2}$. Given that the SGB 1 is more luminous than the SGB 2, we take it as the main branch of the Sgr trailing tail and chose to match its distance to that previously detected using the RC. Tables 1 and 2 present the distance modulus for the SGB detections in both Sgr trailing branches, for each $\Lambda$ bin, and the total errors calculated as described above.

\subsection{Sgr trailing debris with BHB stars}

The Sgr trailing debris sub-structure discovered above ought to be visible with tracers other than SGB stars. Consequently, we slice through the stream using BHB stars. The latter have long been the working horse of Galactic stellar halo studies thanks to their unique properties: low levels of contamination, well-calibrated absolute magnitudes and robust identification by means of multi-band photometry only (see e.g. Yanny et al. 2000; Clewley et al. 2002; Newberg et al. 2003; Sirko et al. 2004; Xue et al. 2008; Bell et al. 2010; Deason et al. 2011; Ruhland et al. 2011; Deason et al. 2012, 2014; Belokurov et al. 2014; Belokurov \& Koposov 2016). As mentioned earlier, VST ATLAS provides ugriz measurements, therefore BHB stars can be selected with the $u-g, g-r$ cuts similar to those normally used for the SDSS photometry, as explained in the literature referenced above. In practice, given that the VST ATLAS $u$ band does not match the SDSS $u$ exactly, the BHB selection boxes differ slightly between the two surveys. More precisely, we use the simplified version of the BHB color cuts, i.e. for the SDSS: $1<u-g<1.5,-0.35<g-r<-0.05$, and $0.9<u-g<1.3,-0.35<g-r<-0.05$ for the VST ATLAS. We assign distances to the candidate BHB stars using the absolute magnitude calibration of Belokurov \& Koposov (2016) which is almost identical (in terms of the absolute magnitude behavior as a function of the $g-r$ color) to that of Deason et al. (2011).

2 Note that we offset the distances by a small amount to improve agreement with the measurement based on the RR Lyrae stars as described in Section 3.4. 
Table 2. Distance modulus to the trailing SGB 1 and SGB 2 peaks in the SGB luminosity function along the Sgr faint stream

\begin{tabular}{lcccccc}
\hline \hline $\begin{array}{l}\tilde{\Lambda}_{\odot} \\
\left(^{\circ}\right)\end{array}$ & $\begin{array}{c}\mathrm{m}-\mathrm{M}(\mathrm{SGB} 1 \mathrm{a}) \\
(\mathrm{mag})\end{array}$ & $\begin{array}{c}\sigma(\mathrm{m}-\mathrm{M}) \\
(\mathrm{mag})\end{array}$ & $\begin{array}{c}\mathrm{m}-\mathrm{M}(\mathrm{SGB} 1 \mathrm{~b}) \\
(\mathrm{mag})\end{array}$ & $\begin{array}{c}\sigma(\mathrm{m}-\mathrm{M}) \\
(\mathrm{mag})\end{array}$ & $\begin{array}{c}\mathrm{m}-\mathrm{M}(\mathrm{SGB} 2) \\
(\mathrm{mag})\end{array}$ & $\begin{array}{c}\sigma(\mathrm{m}-\mathrm{M}) \\
(\mathrm{mag})\end{array}$ \\
\hline-77.5 & 16.84 & 0.06 & 17.48 & 0.13 & 15.97 & 0.09 \\
-75.5 & 17.00 & 0.14 & 17.37 & 0.11 & & \\
-67.5 & 16.88 & 0.12 & 17.48 & 0.06 & & \\
-62.5 & 16.69 & 0.10 & 17.34 & 0.09 & 15.99 & 0.16 \\
-57.5 & 16.56 & 0.08 & 17.51 & 0.25 & 15.79 & 0.08 \\
-52.5 & 16.83 & 0.13 & 17.45 & 0.11 & 15.84 & 0.19 \\
-47.5 & 16.71 & 0.12 & 17.39 & 0.06 & 15.83 & 0.09 \\
-42.5 & 16.89 & 0.07 & 17.47 & 0.03 & & \\
-37.5 & 16.94 & 0.05 & 17.52 & 0.08 & 15.97 & 0.08 \\
\hline
\end{tabular}

The top row of Figure 8 gives the density of the candidate BHB stars along the line of sight as a function of $\tilde{\Lambda}_{\odot}$. Here we extend the range of Sgr longitude further away from the progenitor to be able to reproduce the previous Sgr debris detections from e.g. Koposov et al. (2012). Bear in mind that the BHB selection employed aims to maximize completeness of BHB stars, at the cost of an higher (compared to more sophisticated color-color selection boxes) contamination. Typical contaminants for us to consider are the Blue Stragglers (BS) and MSTO stars. These, however, are fainter by $\sim 1.7 \mathrm{mag}$ and can be easily identified as a broad "shadow" to the narrow BHB sequence. The top left panel of the Figure explores the range of $-8^{\circ}<\tilde{\mathrm{B}}_{\odot}<0^{\circ}$, appropriate for the bright branch of the Sgr trailing tail. The counts in each column have been normalized by the column's total to emphasize the strongest overdensities at each $\tilde{\Lambda}_{\odot}$. The two solid lines show the distance gradient of the Sgr and Cetus stream. From $\tilde{\Lambda}_{\odot}=-140^{\circ}$ to $-30^{\circ}$ the BHBs associated with the Sgr stream proceed from $\mathrm{m}-\mathrm{M} \sim 17.6 \mathrm{mag}$ to $\sim 17.0 \mathrm{mag}$. On the contrary, the heliocentric distance of the BHBs in the Cetus stream increases with $\tilde{\Lambda}_{\odot}$. The streams reach the same distance modulus at $\tilde{\Lambda}_{\odot}=-100^{\circ}$. In the region studied here, and in particular near the Sgr dwarf core, the Cetus stream is detected more than 1 mag further away than the Sgr. The strongly different distant gradients discard the possibility that some of our detections could belong to the CPS.

Starting at large distances from the Sgr dwarf, at $-140^{\circ}<\tilde{\Lambda}_{\odot}<-100^{\circ}$, the narrow dark stripe corresponding to the overdensity of BHB stars can be seen at $17<m-M<18$. It is followed by a broader BS "shadow" at $m-M>19$. Both BHB and BS sequences show a shallow distance gradient with $\tilde{\Lambda}_{\odot}$. Around $-100^{\circ}<\tilde{\Lambda}_{\odot}<-70^{\circ}$, the Sgr stream overlaps with another stellar halo sub-structure, the Cetus Polar Stream (CPS; Newberg et al. 2009). As demonstrated in Koposov et al. (2012), on the sky, the Cetus stream runs at an angle with respect to the track of the Sgr trailing tail. Along the line of sight, the Cetus stream follows a distance gradient opposite to that of the Sgr trailing debris. At $-100^{\circ}<\tilde{\Lambda}_{\odot}<-30^{\circ}$, the Sgr trailing tail continues along the distance gradient seen at higher $\left|\tilde{\Lambda}_{\odot}\right|$, but is now clearly much broader than its continuation further down the stream or compared to Cetus. In fact, hints of two distinct sequences can be discerned.

To scrutinize the line-of-sight sub-structure of the Sgr trailing tail, the lower row of Figure 8 shows 1D slices through the $2 \mathrm{D}$ density distributions given in the top row. In particular, the bottom left panel displays the histogram of stars in the bright branch, i.e. at negative $\tilde{\mathbf{B}}_{\odot}$ in the restricted range of $-55^{\circ}<\tilde{\Lambda}_{\odot}<-30^{\circ}$. Two distinct bumps are noticeable: a narrow one with a peak at $m-M \sim 16.3$ and a broad one with the maximum at $m-M \sim 16.9$. These appear to be in perfect agreement with the distance measurements based on the SGB analysis reported in Table 1 for the same range of $\tilde{\Lambda}_{\odot}$. Stream 1 contains $\sim 150$ stars, while the "background" of the distribution has $\sim 60$ stars, consistent with a peak significance $>10 \sigma$. For Stream 2 the significance is $\sim 5 \sigma$, with an estimated background of 24 stars and $26 \mathrm{BHB}$ stars in excess.

Similarly, the right column of Figure 8 presents the behavior of the density of the candidate BHB stars in the faint branch of the trailing tail. Here, features similar to those in the left column are observable, albeit with some curious differences. For example, only a small portion of the Cetus stream can be seen at $-100^{\circ}<\tilde{\Lambda}_{\odot}<-80^{\circ}$. This is due to the misalignment between CPS and Sgr, with the former moving down to lower declinations. Note that the counterparts of both SGB 1 (more luminous component, at $m-M \sim 17$ ) and SGB 2 (less prominent overdensity, at $m-M \sim 16$ ) can be discerned. Interestingly, Stream 1 appears to break up into further two sub-structures, again in agreement with the complex signal in the SGB residuals map presented above. The significance of the two sub-structures in Stream 1 is $\sim 3 \sigma$ each, i.e. twice as significant as their SGB counterparts (see Section 2.1). In particular, the peak at $m-M \sim 17.2 \mathrm{mag}$ has 43 stars while there are 26 in the background. The nearest component, at $m-M \sim 16.8 \mathrm{mag}$, has $57 \mathrm{BHBs}$ (with $\sim 38$ stars in the estimated background). Considering the contribution of both peaks, as one single detection, the number of BHBs is 150 , with a background level of $\sim 75$ stars, giving an overall significance greater than $10 \sigma$. The stream 2 has a $4 \sigma$ significance (20 stars in excess, over an estimated background of $20 \mathrm{BHBs})$.

Overall, the BHB stars fully corroborate the picture established earlier with the SGB tracers: the trailing debris appears to be bifurcated into two components along the line of sight. Although, he bright Sgr trailing arm detections appear to be much more significant compared to the ones found at the faint arm. Note, however, that without kinematic information it is impossible to establish with $100 \%$ certainty that the brighter BHBs have indeed originated from the Sgr dwarf. Perhaps, these could belong to a different halo substructure, unrelated to Sagittarius. To clarify further the nature of the BHB stars with lower $m-M$, their position on the sky is studied in Section 4.3. 
Table 3. Distance modulus peaks of RRL stars along Sgr trailing tail

\begin{tabular}{lcccc}
\hline \hline $\begin{array}{l}\tilde{\Lambda}_{\odot} \text { Sgr } \\
\left({ }^{\circ}\right)\end{array}$ & $\begin{array}{c}\text { m-M (RRL 1) } \\
(\mathrm{mag})\end{array}$ & $\begin{array}{c}\sigma(\mathrm{m}-\mathrm{M}) \\
(\mathrm{mag})\end{array}$ & $\begin{array}{c}\mathrm{m}-\mathrm{M}(\mathrm{RRL} 2) \\
(\mathrm{mag})\end{array}$ & $\begin{array}{c}\sigma(\mathrm{m}-\mathrm{M}) \\
(\mathrm{mag})\end{array}$ \\
\hline-44.38 & 16.87 & 0.08 & 16.40 & 0.19 \\
-33.13 & 16.82 & 0.14 & 16.28 & 0.27 \\
-21.88 & 16.90 & 0.04 & & \\
-10.63 & 17.11 & 0.02 & & \\
\hline
\end{tabular}

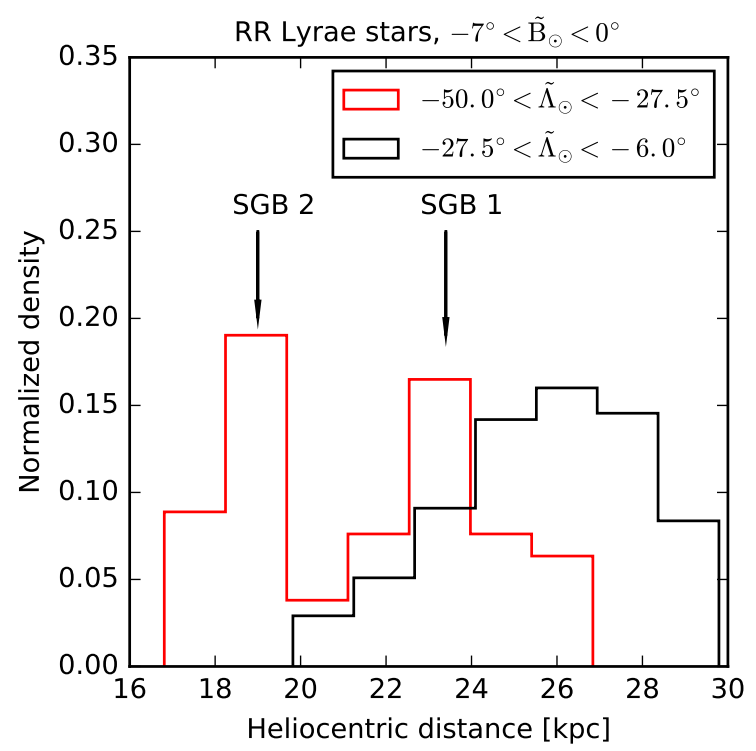

Figure 9. Heliocentric distances for RRL stars in Sgr 1, restricted to the Sgr bright arm region (i.e., $-7^{\circ}<\tilde{\mathrm{B}}_{\odot}<0^{\circ}$ ). The red histogram corresponds to RRL stars located at $-50^{\circ}<\tilde{\Lambda}_{\odot}<-27.5^{\circ}$ and the black histogram to those located at $-27.5^{\circ}<\tilde{\Lambda}_{\odot}<-6.0^{\circ}$. The distribution of RRL stars located near the edge of VST detections, further away from Sgr dwarf, is bimodal, with two peaks consistent with the detections of SGB and BHB stars from VST ATLAS survey. For the innermost region, where VST does not observe, the RRL stars have a distribution consistent with only one main peak, but wider than the previous detections.

\subsection{Sgr trailing tail with CRTS RR Lyrae}

RR Lyrae (RRL) are old (at least 10 Gyr old) and metalpoor pulsating stars. RRL are excellent distance indicators and indispensable tracers of sub-structure in the halo (see e.g., Catelan 2009; Catelan \& Smith 2015, and references therein). However, they are much less numerous compared to other stellar tracers, such as MSTO, SGB and often even BHB stars, and generally require variability surveys to be identified. RRL data from previous wide-field and dedicated surveys have been used to trace the Sgr stream. These include the SDSS Stripe 82 (Ivezić et al. 2000; Watkins et al. 2009), QUEST (Vivas et al. 2005), and the SEKBO survey (Prior et al. 2009). The datasets above have mostly focused on the Northern sky, at declinations generally higher than $-10^{\circ}$, thus missing the portion of the Sgr stream closest to the progenitor.

The Catalina Real-Time Transient Survey (CRTS; Larson et al. 2003; Drake et al. 2009) has filled the gap in the
RRL map of the Milky Way, detecting variable stars across the Southern hemisphere, reaching as low as $-75^{\circ}$ in declination. Recently, Torrealba et al. (2015) identified several promising overdensities in the Southern sky using RRL stars from CRTS. The most significant of these overdensity candidates is dubbed Sgr 1, with a $>15 \sigma$ significance. Based on its $3 \mathrm{D}$ position, Sgr 1 is very likely a part of the trailing tail of the Sgr dwarf in the South. The Sgr 1 sub-structure contains 327 RRL stars, assuming a model for the expected number of RRL in the halo at that position on the sky (for details, see Torrealba et al. 2015). Clearly, with a substantial tally of 327 RRL stars, Sgr 1 more than fills in the gap in the Sgr stream coverage in the vicinity of the progenitor.

From the 327 RRL stars originally identified in the Sgr 1 overdensity, we selected only those confined to the plane of the bright trailing arm of Sgr, i.e. between $-7^{\circ}<\tilde{\mathrm{B}}_{\odot}<0^{\circ}$. This spatial selection reduces the the sample size to 250 RRL, spanning the longitude of the Sgr stream from $\tilde{\Lambda}_{\odot}=$ $-50^{\circ}$ to $\tilde{\Lambda}_{\odot}=-6^{\circ}$. Unfortunately, because of the spatial limits of the survey, there are only 42 RRL located at positive $\tilde{\mathrm{B}}_{\odot}$, thus making it unfeasible to track down the peaks for the faint Sgr branch. Therefore, the RRL distribution is only analyzed here for the bright branch of the Sgr trailing arm.

To trace the distance gradient in RRL stars, the length of the Sgr 1 overdensity was divided into 4 bins, each $11.25^{\circ}$ wide, similar to the SGB analysis. The wider $\tilde{\Lambda}_{\odot}$ bins are chosen to ensure enough RRL stars in each bin. The RRL number density along the stream shows a strong gradient: indeed, the bins further away from the main body of Sgr are less populated, by a factor of two, compared to the two innermost bins (where there are more than 70 RRL stars per bin). For each $\tilde{\Lambda}_{\odot}$ bin, the heliocentric distance distribution was modeled with either one or two Gaussians. The heliocentric distances come directly from the catalogue published by Torrealba et al. (2015), and they are accurate to $\sim 10 \%$ (depending on the magnitude of the RRL, the distance uncertainty is between $7 \%$ and $12 \%$, see Drake et al. 2013a). We detect two peaks in the heliocentric distance distribution of RRL in Sgr 1 in the first two bins, namely at $\tilde{\Lambda}_{\odot} \sim-40^{\circ}$ and $\tilde{\Lambda}_{\odot} \sim-33^{\circ}$. In the two bins closest to the progenitor, the distance distribution of RRL is consistent with one broad peak which was modeled with a single Gaussian. Figure 9 shows the heliocentric distance distribution for RRL stars in Sgr 1 located at $-50.0^{\circ}<\tilde{\Lambda}_{\odot}<-27.5^{\circ}$ (red histogram) and at $-27.5^{\circ}<\tilde{\Lambda}_{\odot}<-6.0^{\circ}$ (dotted line histogram). RRL stars further away from the Sgr dwarf (i.e., at higher $\left|\tilde{\Lambda}_{\odot}\right|$ ) show a clear bimodality, with two peaks located at $\sim 19.3$ and $23.4 \mathrm{kpc}$, nicely connecting the two peaks found using SGB and BHB stars. Closer to the dwarf, the distribution peaks at $\sim 26.0 \mathrm{kpc}$, with a wider range of distances. This suggests that most probably the two peaks forming the fork get merged as they are going towards the actual position of the Sgr dwarf nucleus. The gap between both distributions, at $\sim 20 \mathrm{kpc}$, is mostly due to selection effects as the sample of RRL stars from Sgr 1 was selected based on the local overdensity of RRL stars with respect to the density of RRL stars in the halo (Torrealba et al. 2015). In the figure, we adopted the same naming convention for the two peaks with most distant dubbed RRL 1 while the nearest one is RRL 2.

As the RRL stars are the most trustworthy distance indicators at our disposal, we can tighten up the SGB absolute 

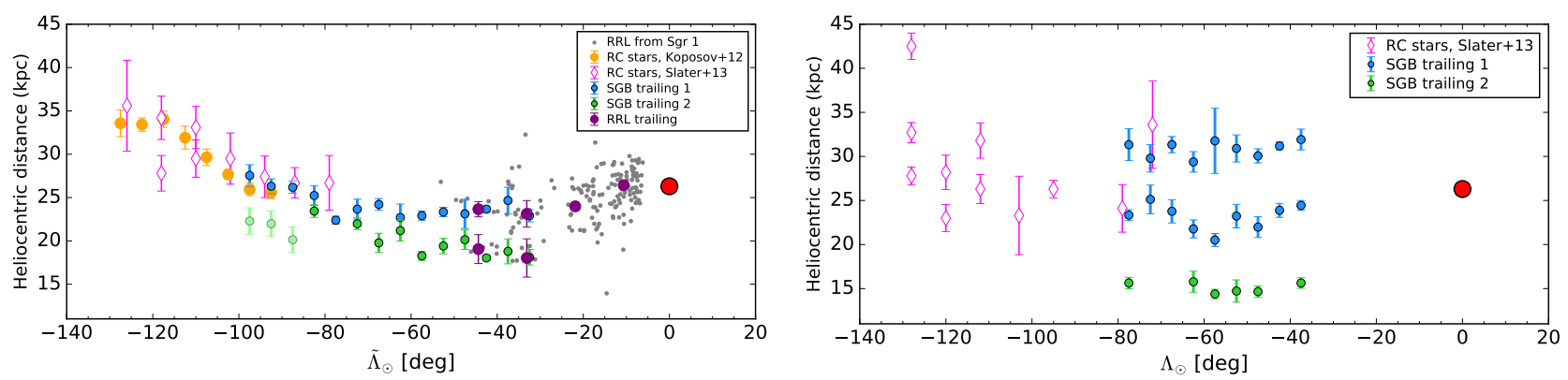

Figure 10. Left: Heliocentric distances as a function of the longitude along the stream. Yellow circles correspond to the RC measurements by Koposov et al. (2012), while open pink rhombs are the RC detections of Slater et al. (2013) using the Pan-STARRS data. The SGB data-points obtained in this work are marked with blue (trailing 1) and green (trailing 2) circles. Peaks in the RRL distribution (using the Sgr 1 sample of Torrealba et al. 2015) are marked with purple circles, while the individual RRL stars from Sgr 1 are marked with grey points. The position of the Sgr dwarf, at $\tilde{\Lambda}_{\odot}=0^{\circ}$, and heliocentric distance of $26.3 \mathrm{kpc}$, is marked with a filled red circle. Right: Heliocentric distances as a function of $\tilde{\Lambda}_{\odot}$ for the faint branch of the trailing arm (located at $\tilde{\mathrm{B}}_{\odot}>0^{\circ}$ ). Open diamonds correspond to the RC detections from Slater et al. (2013). Measurements based on the SGB stars are marked with blue (trailing 1) and green (trailing 2) circles. The main stream, trailing 1 , splits into two parallel sequences, separated by $\sim 5 \mathrm{kpc}$ in heliocentric distance.

distance scale. More precisely, using the RC distances from Koposov et al. (2012) as our reference point (see Section 3.2), there appears to be a small offset of $\sim 0.3 \mathrm{kpc}$ compared to the distances of RRL at $\tilde{\Lambda}_{\odot}=-45^{\circ}$. Therefore, we choose to match the SGB Sgr stream distances at $\tilde{\Lambda}_{\odot}=-44^{\circ}$ to those dictated by the RRL, i.e. $23.7 \mathrm{kpc}$. Indeed, an impressive match (as can be judged from Fig. 10 below) between the two overdensities detected with SGB and RRL stars obtains as a result, when we offset the distances at $\tilde{\Lambda}_{\odot}=-97.5$ and -92.5 by 0.4 and $2 \mathrm{kpc}$, respectively, as compared to the Koposov et al. (2012) scale. The distance modulus to each of the peaks detected as well as the errors (derived as in the case of the SGB stars) are reported in Table 3.

\section{DISCUSSION AND CONCLUSIONS}

\subsection{Distances along the Sgr stream}

Figure 10 presents the heliocentric distance evolution along the Sgr trailing arm as a function of $\tilde{\Lambda}_{\odot}$, as measured with different stellar tracers. Included in the Figure are the previous detections, such as the RC measurements of Koposov et al. (2012) shown as filled orange circles, as well as the measurements based on the SGB stars (green and blue circles) carried out here. The left panel of the Figure shows the distance measurements for the bright component of the trailing arm, i.e. for stars with $\tilde{\mathrm{B}}_{\odot}<0^{\circ}$, and additionally includes the Pan-STARRS RC measurements by Slater et al. (2013), shown as open pink rhombs; as well as the individual RRL stars (small grey dots) and their mean distances in bins of $\tilde{\Lambda}_{\odot}$ (filled lilac circles).

The right panel of Figure 10 gives the distances along the faint trailing stream branch, i.e. that at $1^{\circ}<\tilde{\mathrm{B}}_{\odot}<9^{\circ}$. The "SGB trailing 1" detections correspond to the most prominent SGB peaks found in each $\tilde{\Lambda}_{\odot}$ bin. Unlike the primary SGB signal found in the Sgr bright arm (see the left panel of the Figure), this component appears to split into two, almost parallel, branches separated by $\sim 5 \mathrm{kpc}$. Curiously, there are hints of the two streams in the previous measurements of Slater et al. (2013). However, that work lacked the appropriate sky coverage to trace the stream over a long
$\tilde{\Lambda}_{\odot}$ range. As discussed in the previous Section, at $\sim 15 \mathrm{kpc}$, there appear strong hints of another, albeit less prominent sub-structure, dubbed "SGB trailing 2", which we believe is the counterpart of the secondary SGB detection at similar distances in the bright branch. Note that the multiple SGB detections along the line of sight are corroborated by the BHB star counts as a function of distance modulus. For example, the three substructures found in the faint Sgr branch are also recovered using BHB stars (see the right panel of Figure 8), where two prominent peaks are found at m-M 16.8 and $17.3 \mathrm{mag}$ (corresponding to the two trailing SGB 1 ), while a less prominent peak is found at $\mathrm{m}-\mathrm{M} \sim 16.1 \mathrm{mag}$ (SGB 2). Unfortunately, the number of CRTS RRL stars at $\tilde{\mathrm{B}}_{\odot}>0^{\circ}$ is not sufficient to trace the faint Sgr stream at this stage.

Interestingly, the heliocentric distances reached by the most distant SGB 1 debris in the faint Sgr branch are similar to the distances reported for the CPS by Newberg et al. (2009); Yam et al. (2013). While superficially these detections might appear related, as shown in Figure 8, the orbit of the CPS is not consistent with the track of these substructures (see also Figure 8 from Slater et al. 2013).

\subsection{In the Sgr orbital plane}

Figure 11 displays the projection of the Sgr stream detections onto the Sgr orbital plane (see Belokurov et al. 2014, for more details). Also shown as the underlying greyscale density is the distribution of the Sgr particles from the numerical model of Law \& Majewski (2010). As is obvious from the left panel of the Figure, there is good agreement between the SGB 1 distance measurements within the bright branch of the trailing arm (shown in blue) and the simulation. The sequence of the SGB 1 detections and its continuation with RRL (lilac) point directly towards the Sgr dwarf. In comparison, the SGB 2 sub-structure does not have an obvious counterpart in the Law \& Majewski (2010) simulation. The two stream components -SGB 1 and SGB 2 - start to diverge at $X_{\mathrm{Sgr}} \sim 0 \mathrm{kpc}$, and as indicated by the detections within the VST ATLAS, the SGB 2 structure does not seem to be going towards the Sgr remnant. Curiously, the 

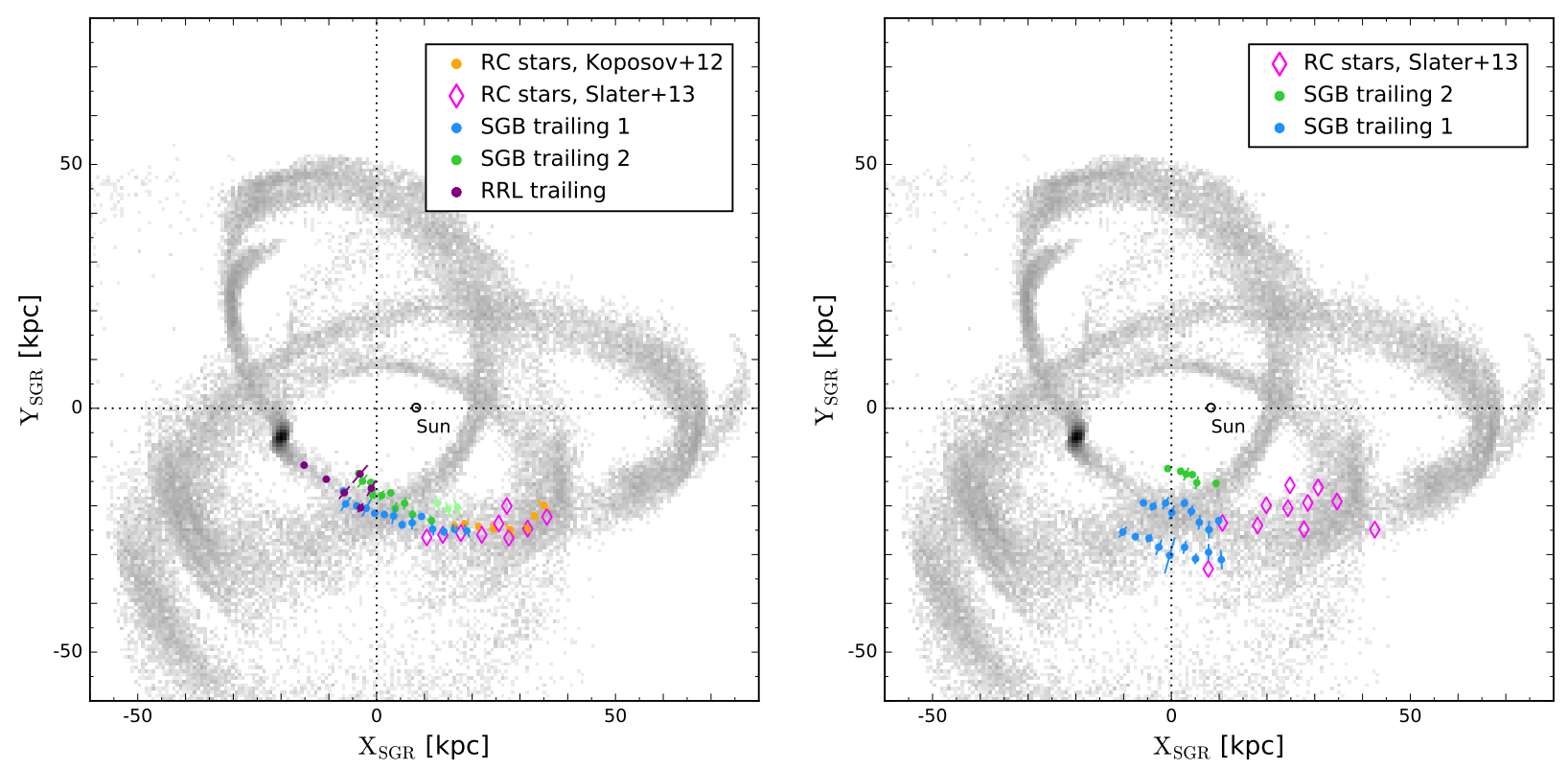

Figure 11. Centroids of the SGB detections, projected on the plane of the Sgr orbit, with the bright (faint) branch shown in the left (right) panel. The pole of the plane is at Galactocentric coordinates $l_{G C}=275^{\circ}$ and $b_{G C}=-14^{\circ}$. The symbols are the same as in Figure 10, but the individual RRL stars from Sgr 1 are not shown here. The particles from the Sgr disruption model by Law \& Majewski (2010) are shown as grey-scale density.

two components diverge at the point where the young trailing debris cross the wrap of the leading tail. This, however, might be a pure coincidence given that the simulated leading tail appears to be moving almost perpendicular to the SGB 2.

Similarly, the right panel of Figure 11 compares the orbital plane distribution of the simulated Sgr debris with the SGB detections of the faint branch of the trailing tail. The most prominent of the two SGB 1 detections in the faint arm, located at the heliocentric distance of $\sim 25 \mathrm{kpc}$, lies on top of the simulated trailing arm and points directly towards the Sgr dwarf nucleus. It connects with the previous detections made by Slater et al. (2013), extending the portion of the stream traced by an additional $\sim 30 \mathrm{kpc}$ or 40 degrees. The other two SGB detections do not appear in the distribution of the simulated debris. However, again, around the location studied here, there appears to be a large spray of the leading debris.

The projection of the Sgr stream detections onto the Sgr's orbital plane conceals the extension of the bright/faint streams below/above the plane in kpc. To clarify, the detections of the bright branch, confined to $-7^{\circ}<\tilde{\mathrm{B}}_{\odot}<0^{\circ}$, correspond to $Z_{\mathrm{SGR}}>-3.5$ and $-2.9 \mathrm{kpc}$ for SGB 1 and 2 , respectively. SGB $1 \mathrm{a}, 1 \mathrm{~b}$ and 2 , in the faint branch, have $Z_{\mathrm{SGR}}$ up to $3.9,5.0$ and $2.5 \mathrm{kpc}$ above the Sgr's plane (equivalent to $1^{\circ}<\tilde{\mathrm{B}}_{\odot}<9^{\circ}$ ). In all the cases, the detections are confined to less than $5 \mathrm{kpc}$ (in absolute value), which is a short distance considering the extension covered in the Sgr's plane coordinates $\mathrm{X}_{\mathrm{SGR}}$ and $\mathrm{Y}_{\mathrm{SGR}}$.

\subsection{Adding the third dimension}

So far, we have limited our study to the two-dimensional slices of the stream, i.e. we have only analyzed the stellar de- bris densities in the plane of the distance and the stream longitude. This, of course, is a necessity created by the complete overlap of the structures we have discovered on the celestial plane. Figure 12 presents an attempt to discern whether the more distant overdensity (SGB 1) and the closer one (SGB 2) behave differently as a function of the Sgr latitude $\tilde{\mathbf{B}}_{\odot}$. For our experiment, we have chosen the piece of the trailing arm where the number counts in the SGB 2 are at the highest level, i.e. $-55^{\circ}<\tilde{\Lambda}_{\odot}<-34^{\circ}$. Furthermore, we have split this section on the stream into two portions shown in the left and the middle panels of the Figure. The selection boundaries used to pick candidate stars belonging to the SGB 1 and SGB 2 sub-structures are shown in the inset of the middle panel of the Figure. In addition to the magnitude-longitude cuts, all stars are also required to have colors consistent with the SGB population, namely $0.52<g-i<0.6$.

According to Figure 12, there is a dramatic increase in the number counts of stars in the SGB boxes from $\tilde{\Lambda}_{\odot}=-55^{\circ}$ to $\tilde{\Lambda}_{\odot}=-34^{\circ}$, i.e. from the left to the middle panel. Most of the density hike is associated with the increase in the contaminating foreground population, but there appears to be also an increase in the stream star counts. Note that while in the right panel of Figure 5 the SGB 1 and SGB 2 overdensities have similar strength, in the histograms presented in Figure 12 the SGB 1 signal (shown in red) is clearly stronger. This is because here the foreground counts were not subtracted. The foreground contamination increases with apparent magnitude, thus causing the SGB 1 signal to be elevated compared to SGB 2. There exist obvious peaks in both the SGB 1 and the SGB 2 distributions, with locations close to $\tilde{\mathrm{B}}_{\odot} \sim 0^{\circ}-$ i.e. in the vicinity of the Sgr orbital plane - thus supporting the hypothesis that both structures detected here used to belong to the Sgr dwarf proper.

Perhaps, the most interesting feature of the Figure is 
the difference in the latitudinal behavior of the two substructures at $-40^{\circ}<\tilde{\Lambda}_{\odot}<-34^{\circ}$ displayed in the middle panel. The more distant SGB 1 appears to have a fairly narrow peak at $\tilde{\mathrm{B}}_{\odot}=2.5^{\circ}$. This can be compared to the black histogram representing the SGB 2 counts, where the still narrow peak is shifted by several degrees towards negative $\tilde{\mathrm{B}}_{\odot}$. One possible, albeit tentative, explanation of the difference in the latitudinal profiles is that SGB 1 connects directly to the progenitor while SGB 2 might not. Note that there is another, broader peak in the distribution of the SGB 2 candidate stars, at $\tilde{\mathrm{B}}_{\odot} \sim 10^{\circ}$. Further away from the remnant, i.e. at $-55^{\circ}<\tilde{\Lambda}_{\odot}<-40^{\circ}$, no discernible difference can be seen in the stellar density profiles of the two sub-structures.

Finally, the right panel of Figure 12 presents the $\tilde{\mathbf{B}}_{\odot}$ density profile of structures traced by BHBs with different distance moduli. Following the above notation, red dotted line corresponds to the main (SGB 1, or $16.6<m-M<17.5$ ) component and solid black to the closer (SGB 2, or $15.9<$ $m-M<16.5)$ one. As expected, the red line traces a broad bump, centered around $\tilde{\mathrm{B}}_{\odot}=0^{\circ}$. The black histogram displays a peak slightly off $\tilde{\mathrm{B}}_{\odot}=0^{\circ}$ location, at around $\tilde{\mathrm{B}}_{\odot}=-3^{\circ}$, similarly to the SGB 2 profile shown in the middle panel of the Figure. Given that there is a very good match between the peak positions and the widths of the density distributions of the bright SGB stars (SGB 2) and the bright BHBs, we conclude that it is likely that both sets of tracers correspond to the same halo structure. Furthermore, taking into account the proximity of the density peaks to $\tilde{\mathbf{B}}_{\odot}=0^{\circ}$, it is very likely that this structure is related to the Sgr disruption.

\subsection{Metallicity of the detected sub-structures}

RRL stars from the catalogue of Torrealba et al. (2015) have photometrically derived metallicities available, thus giving us an opportunity for a straightforward comparison of the chemical properties of the two stream components. As usual, the metallicities of the individual RRL stars were derived using the light curve shape information. The RRL-based $[\mathrm{Fe} / \mathrm{H}]$ distribution of a nearby piece of the trailing tail was studied before by Watkins et al. (2009), who reported a mean metallicity of $[\mathrm{Fe} / \mathrm{H}]=-1.43$ with a dispersion of 0.3 dex. For the portion of the tail covered by the CRTS, we measure average metallicity of $[\mathrm{Fe} / \mathrm{H}]=-1.47$, with a dispersion of $\sigma=0.36$ dex. Clearly, our estimate of the Sgr 1 mean metallicity is very similar to the abundance of the Sgr tail covered by the SDSS Stripe 82, as derived by Watkins et al. (2009). However, it remains uncertain how much of this agreement is due to a genuine similarity of the two portions of the stream studied and how much of it may be due to the crudeness of the RRL photometric $[\mathrm{Fe} / \mathrm{H}]$ scale.

An indication that the agreement may not be fortuitous is provided by the spectroscopic follow-up study of the Sgr trailing RRL stars detected by the SEKBO survey as reported by Prior et al. (2009). Here, a more metal-poor mean abundance of $[\mathrm{Fe} / \mathrm{H}]=-1.79 \pm 0.08$ was found with a dispersion of 0.38 dex. Therefore, spectroscopic metallicities for RRL stars belonging to Sgr 1 are needed to confirm if they are indeed more metal-rich than dictated by the findings of Prior et al. (2009). Note, however that our mean metallicities are in very good agreement with those found for a nearby portion of the stream in the most recent spectroscopic study of Gibbons et al. (2016a). They report a bimodal MDF, in which the more prominent metal-poor component has the mean at -1.33 and a dispersion of 0.27 dex.

Additionally, we have compared the mean metal abundances of the two RRL overdensities detected in the first two $\tilde{\Lambda}_{\odot}$ bins. It turns out that the two overdensities have the same mean metal abundances: at $\tilde{\Lambda}_{\odot}=-43.5^{\circ},[\mathrm{Fe} / \mathrm{H}]$ $=-1.53$ (with dispersion $\sigma=0.2 \mathrm{dex}$ ) for the $9 \mathrm{RRL}$ at 19.2 $\mathrm{kpc}$, while the $11 \mathrm{RRL}$ at $\sim 24 \mathrm{kpc}$ have $[\mathrm{Fe} / \mathrm{H}]=-1.52(\sigma$ $=0.3 \mathrm{dex})$; at $\tilde{\Lambda}_{\odot}=-31.5^{\circ}, 9 \mathrm{RRL}$ at $18 \mathrm{kpc}$ have $[\mathrm{Fe} / \mathrm{H}]$ $=-1.51(\sigma=0.4 \mathrm{dex})$, while the $12 \mathrm{RRL}$ at $22 \mathrm{kpc}$ have $[\mathrm{Fe} / \mathrm{H}]=-1.49(\sigma=0.3 \mathrm{dex})$. It appears therefore that both line-of-sight components detected in the bright Sgr trailing branch are consistent with the metallicities expected for the Sgr stars. Clearly, more information, such as radial velocities of the RRL stars in each of the peaks, would be required to confirm the components as coherent and related structures. Such information will be provided by our team in a forthcoming study (Duffau et al., in preparation).

\subsection{Conclusions}

In this work, we have examined a large portion - approximately $65^{\circ}$ - of the Sgr trailing stream available in the imaging data from the VST ATLAS survey. Most of the area at least $40^{\circ}$ - covered in our analysis has not been studied before using photometric data of such depth. This section of the stream is of particular interest as it is situated in the proximity of the Sgr remnant, i.e. only $40^{\circ}$ from the center of the dwarf. Taking advantage of the depth of the VST ATLAS photometry, we chose to use the SGB population as the main halo sub-structure tracer.

Curiously, at many locations along the Sgr stream studied here, at least two peaks of SGB stars are detected along the line of sight. At its highest, the separation between the peaks is $\sim 0.5 \mathrm{mag}$ (or $\sim 5 \mathrm{kpc}$ at the distance of the stream). However, importantly, we detect a significant variation in the peak separation as a function of the stream longitude $\tilde{\Lambda}_{\odot}$. Therefore, we believe that the secondary SGB detection is not due to a complex co-distant stellar population mix in the stream, but rather reveals the presence of (at least) two distinct sub-structures projected within the Sgr orbital plane. This discovery is reminiscent of the detection of the so-called Branch C behind the Sgr leading tail presented in Belokurov et al. (2006).

We have compared, where possible, the absolute and the relative SGB distance measurements with the previously published values such as those by Koposov et al. (2012) and Slater et al. (2013) and found very good agreement. Furthermore, we have confirmed the authenticity of the SGB lineof-sight detections with other tracers, such as RR Lyrae and BHB stars, and were reassured by an excellent match. The two line-of-sight components are most visible in the bright branch of the Sgr trailing stream, i.e. at $\tilde{\mathrm{B}}_{\odot}<0^{\circ}$. Nonetheless, we also find strong evidence for a similar line-of-sight splitting in the faint branch, i.e. at $\tilde{\mathrm{B}}_{\odot}>0^{\circ}$.

The projection of the VST ATLAS detections onto the Sgr orbital plane reinforces the conclusion that the two substructures are indeed part of the Sgr debris distribution. When viewed in this perspective, the more distant of the two overdensities, the SGB 1, appears to be connecting to the Sgr remnant. The two sub-structures can not be easily 

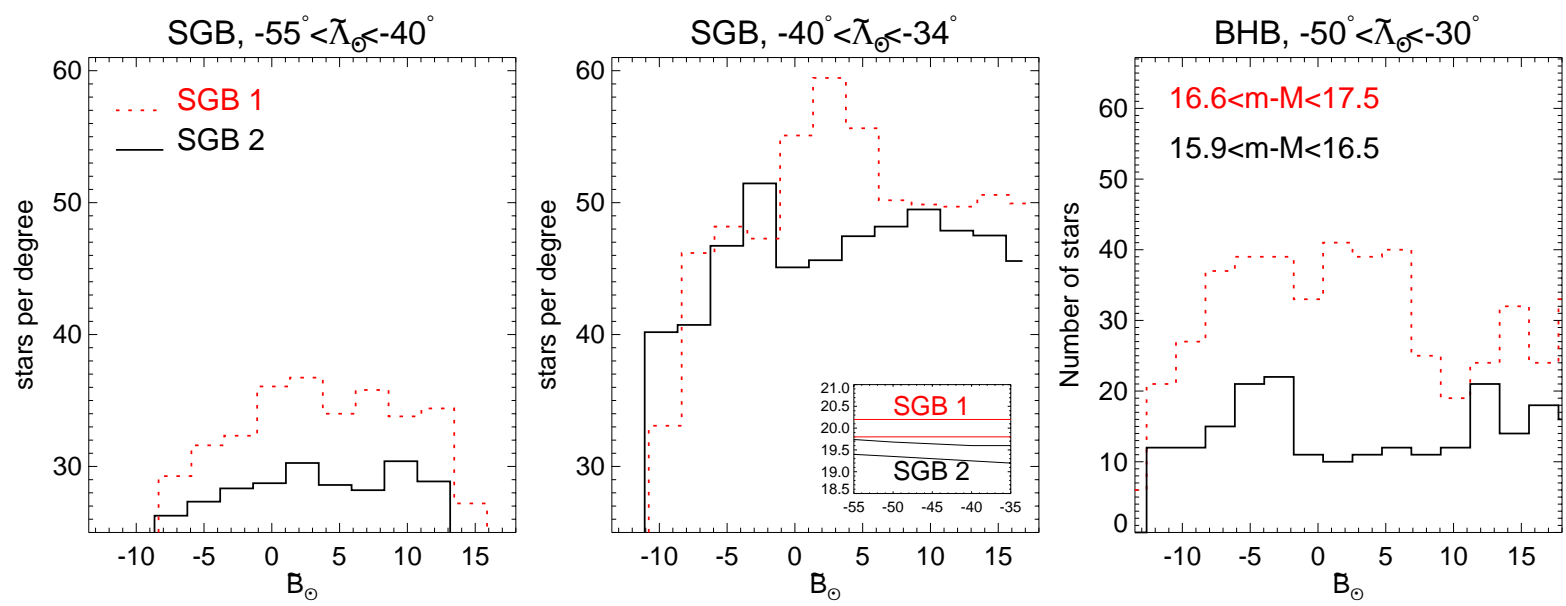

Figure 12. Left and Middle: Density distribution of the candidate SGB stars in the two line-of-sight components (SGB 1 and SGB 2) as a function of the stream latitude $\tilde{B}_{\odot}$. The red dotted (black solid) histograms represent the SGB 1 (SGB 2) component. The SGB stars are selected to have $0.52<(g-i)<0.6$. Additionally, a cut in the space of $i$ magnitude vs. $\tilde{\Lambda}_{\odot}$ is applied and the exact selection boxes are shown in the inset of the middle panel. Note the different locations of the peaks of the two distributions in the middle panel, i.e. the portion of the stream closest to the Sgr remnant. Right: Density distribution of the candidate BHB stars. The main (secondary) structure corresponding to the SGB 1 (SGB 2) component is shown with dotted red (solid black) line. Across the three panels, the solid black and the dotted red profiles show significant peaks around $\tilde{\mathbf{B}}_{\odot}=0^{\circ}$, thus lending support to our hypothesis that the structures we see along the line of sight are confined to the Sgr orbital plane, and therefore are likely connected to the accretion of the Sgr dwarf. Moreover, in the second and third panels, the secondary streams traced by the SGB and the BHB stars (black line) are off-set by a similar amount from zero, i.e. $\sim-5^{\circ}$, thus providing further evidence that the two stellar tracers are picking up the same structure.

separated at large distances from Sgr; however, the presence of an additional stream component is betrayed by the increased width of the line-of-sight distribution of the debris. On approach to the dwarf, however, at $\tilde{\Lambda}_{\odot} \sim-60^{\circ}$, the SGB 2 forks out, and, following a distinct distance gradient, appears to undershoot the Sgr dwarf. In comparison to the simulation of Law \& Majewski (2010), the bifurcation happens around the location where the young trailing debris runs into an old wrap of the leading arm. This poses a question whether the SGB 2 could actually be one of the old wraps of the Sgr stream. Note, however, that there is no obvious counterpart of the SGB 2 sub-structure in the above mentioned simulation; therefore, at the moment, this scenario seems somewhat unlikely.

With the new detections presented here, the current picture of the Sgr tidal tails appears incredibly complex. The trailing arm is ripped apart on the plane of the sky, but additionally shows a bifurcation along the line of sight. If these, previously unseen portions of the Sgr debris were indeed the older wraps of the stream, they would provide yet another confirmation that the Sgr tails are longer than previously thought, and are likely originating from a bigger parent galaxy. Additionally, these new wraps ought to provide a good leverage in constraining the properties of the gravitational potential of the Galaxy. Alternatively, the messy twisting of the tidal tails could be a sign of the complex structure of the progenitor. For example, Peñarrubia et al. (2010) show that line-of-sight stream splitting is possible if the Sgr dwarf was a disc galaxy. Gibbons et al. (2016b) confirms that even in a completely spherical host potential, the discy dwarf disruption will produce sprays of tidal debris in distinct orbital planes. Clearly, to establish the nature of the multiple sub-structures within the Sgr tails, it would help to focus on the part of the stream so far virtually unstudied, namely the section closest to the progenitor.

\section{ACKNOWLEDGEMENTS}

This publication makes use of data products from the AAVSO Photometric All Sky Survey (APASS), funded by the Robert Martin Ayers Sciences Fund and the National Science Foundation.

The research leading to these results has received funding from the European Research Council under the European Union's Seventh Framework Programme (FP/20072013)/ERC Grant Agreement no. 308024.

This project is supported by CONICYT's PCI program through grant DPI20140066. Additional support is provided by the Ministry for the Economy, Development, and Tourism's Iniciativa Científica Milenio through grant IC 120009, awarded to the Millennium Institute of Astrophysics; by Proyecto Fondecyt Regular \#1141141; and by Proyecto Basal PFB-06/2007. C.N. acknowledges support from CONICYT-PCHA grant Doctorado Nacional 201521151643.

\section{REFERENCES}

Ahn C. P., et al., 2012, ApJS, 203, 21

Bell E. F., Xue X. X., Rix H.-W., Ruhland C., Hogg D. W., 2010, AJ, 140, 1850

Belokurov V., Koposov S. E., 2016, MNRAS, 456, 602

Belokurov V., et al., 2006, ApJ, 642, L137

Belokurov V., et al., 2014, MNRAS, 437, 116

Catelan M., 2009, Ap\&SS, 320, 261

Catelan M., Smith H. A., 2015, Pulsating Stars (Wiley-VCH) 
Clewley L., Warren S. J., Hewett P. C., Norris J. E., Peterson R. C., Evans N. W., 2002, MNRAS, 337, 87

Correnti M., Bellazzini M., Ibata R. A., Ferraro F. R., Varghese A., 2010, ApJ, 721, 329

Deason A. J., Belokurov V., Evans N. W., 2011, MNRAS, 416, 2903

Deason A. J., et al., 2012, MNRAS, 425, 2840

Deason A. J., Belokurov V., Koposov S. E., Rockosi C. M., 2014, ApJ, 787, 30

Drake A. J., et al., 2009, ApJ, 696, 870

Drake A. J., et al., 2013a, ApJ, 763, 32

Drake A. J., et al., 2013b, ApJ, 765, 154

Erkal D., Sanders J. L., Belokurov V., 2016a, MNRAS, 461, 1590

Erkal D., Belokurov V., Bovy J., Sanders J. L., 2016b, MNRAS, 463, 102

Gibbons S. L. J., Belokurov V., Evans N. W., 2014, MNRAS, 445, 3788

Gibbons S. L. J., Belokurov V., Evans N. W., 2016a, preprint, (arXiv: 1607.00803 )

Gibbons S. L. J., Belokurov V., Erkal D., Evans N. W., 2016b, MNRAS, 458, L64

Henden A. A., Templeton M., Terrell D., Smith T. C., Levine S., Welch D., 2016, VizieR Online Data Catalog, 2336

Ivezić Ž., et al., 2000, AJ, 120, 963

Koposov S. E., et al., 2012, ApJ, 750, 80

Koposov S. E., Irwin M., Belokurov V., Gonzalez-Solares E., Yoldas A. K., Lewis J., Metcalfe N., Shanks T., 2014, MNRAS, 442, L85

Larson S., Beshore E., Hill R., Christensen E., McLean D., Kolar S., McNaught R., Garradd G., 2003, in AAS/Division for Planetary Sciences Meeting Abstracts \#35. p. 982

Law D. R., Majewski S. R., 2010, ApJ, 714, 229

Majewski S. R., Skrutskie M. F., Weinberg M. D., Ostheimer J. C., 2003, ApJ, 599, 1082

Newberg H. J., et al., 2003, ApJ, 596, L191

Newberg H. J., Yanny B., Willett B. A., 2009, ApJ, 700, L61

Niederste-Ostholt M., Belokurov V., Evans N. W., Peñarrubia J., 2010, ApJ, 712, 516

Peñarrubia J., Belokurov V., Evans N. W., Martínez-Delgado D., Gilmore G., Irwin M., Niederste-Ostholt M., Zucker D. B., 2010, MNRAS, 408, L26

Prior S. L., Da Costa G. S., Keller S. C., 2009, ApJ, 704, 1327

Ruhland C., Bell E. F., Rix H.-W., Xue X.-X., 2011, ApJ, 731, 119

Schlafly E. F., Finkbeiner D. P., 2011, ApJ, 737, 103

Schlegel D. J., Finkbeiner D. P., Davis M., 1998, ApJ, 500, 525

Shanks T., et al., 2015, MNRAS, 451, 4238

Sirko E., et al., 2004, AJ, 127, 899

Slater C. T., et al., 2013, ApJ, 762, 6

Torrealba G., et al., 2015, MNRAS, 446, 2251

Vivas A. K., Zinn R., Gallart C., 2005, AJ, 129, 189

Watkins L. L., et al., 2009, MNRAS, 398, 1757

Xue X. X., et al., 2008, ApJ, 684, 1143

Yam W., Carlin J. L., Newberg H. J., Dumas J., O'Malley E., Newby M., Martin C., 2013, ApJ, 776, 133

Yanny B., et al., 2000, ApJ, 540, 825

Yanny B., et al., 2009, ApJ, 700, 1282

de Boer T. J. L., Belokurov V., Koposov S., 2015, MNRAS, 451, 3489

This paper has been typeset from a $\mathrm{T}_{\mathrm{E}} \mathrm{X} / \mathrm{LA} \mathrm{T} \mathrm{X}$ file prepared by the author. 